\title{
Second-Harmonic Generation from a Quantum Emitter Coupled to a Metallic Nanoantenna
}

\author{
Antton Babaze, ${ }^{*,+\ddagger}$ Ruben Esteban, ${ }^{\ddagger}, \mathbb{I}$ Javier Aizpurua, ${ }^{\dagger} \ddagger$ and Andrei G. Borisov ${ }^{\S}$ \\ +Materials Physics Center CSIC-UPV/EHU, Paseo Manuel de Lardizabal 5 20018, Donostia-San \\ Sebastián, Spain \\ $\ddagger$ Donostia International Physics Center DIPC, Paseo Manuel de Lardizabal 4 20018, \\ Donostia-San Sebastián, Spain \\ IIIKERBASQUE, Basque Foundation for Science, 48013 Bilbao, Spain \\ §Institut des Sciences Moléculaires d'Orsay, UMR 8214 CNRS-Université Paris-Sud, Bât. 520, \\ 91405 Orsay Cedex, France \\ E-mail: anttonbabaze@dipc.org
}

\section{Abstract}

We use time-dependent density functional theory and a semiclassical model to study second-harmonic generation in a system comprising a quantum emitter and a spherical metallic nanoparticle, where the transition frequency of the quantum emitter is set to be resonant with the second harmonic of the incident frequency. The quantum emitter is shown to enable strong secondharmonic generation, which is otherwise forbidden because of symmetry constraints. The time-dependent density functional theory calculations allow one to identify the main mechanism driving this nonlinear effect, where the quantum emitter plays the role of an optical resonator that experiences the nonlinear near fields generated by the metallic nanoantenna located nearby. The influence of the intrinsic properties of the quantum emitter and the nanoantenna, together with the relative position of both in the coupled system, allows for a high degree of control of the nonlinear light emission. The main effects and contributions to this nonlinear process can be correctly captured by a semiclassical description developed in this work.

\section{Keywords}

second-harmonic generation; plasmonics; quantum emitter; nonlinear optical response; electromagnetic coupling

The coupling between incident electromagnetic radiation and collective electronic excitations, so-called surface plasmons, in metallic nanoparticles (MNPs) al- lows one to localize, enhance, and control the near fields around the nanoparticles at scales well below the wavelength of light. The resonant excitation of the plasmonic modes, along with the intrinsic nonlinearity of the metals, results in a strong nonlinear optical response of plasmonic structures with a great variety of applications. $\frac{122}{1 n}$ particular, second-harmonic generation (SHG), whereby two photons at the fundamental frequency are absorbed to emit one photon at the second-harmonic frequency is at the focus of very active research owing to its practical and fundamental interest. ${ }^{3-12}$ For typical plane-wave incidence, the SHG is forbidden for materials and nanostructures that are centrosymmetric. This nonlinear response is thus very sensitive to the geometry of the system and to surface effects that eventually may break the symmetry constraints and lead to the emission of light at the second harmonic. $\left.{ }^{213}-18\right]$ In this context, it has been shown that plasmonic structures resonant at the fundamental or at the second-harmonic frequency (or at both frequencies) can give rise to manyfold enhancement of the SHG. ${ }^{89] 19}$.31 Recent experiments have also shown the polarization-resolved probing of the nonlinear near field distribution of metallic structures by using doubly resonant plasmonic antennas. $\frac{32}{32}$ On the other hand, the coupling of a quantum emitter (QE), such as an organic molecule or a quantum dot with a plasmonic nanoantenna has been widely studied in previous works, analyzing diverse aspects such as surface-enhanced Raman scattering, single-molecule spectroscopy, strong coupling or the effect of electronic conductivity through molecules. 33 Moreover, the capability of the MNPQE interaction to modify the second-harmonic emis- 
sion has been demonstrated for plasmonic nanostructures, $\stackrel{42[43}{,}$ and also the strong nonlinear response of graphene nanostructures has been proposed as a way to excite the electronic transitions in atomic or molecular species. ${ }^{44}$

Here we study the SHG resulting from a hybrid system consisting of a QE placed in the vicinity of a spherical MNP, as scketched in Figure 19. The small individual centrosymmetric nanoparticle does not allow for second-harmonic emission, but the presence of the $\mathrm{QE}$ lifts this symmetry constraint. When the electronic transition frequency of the $\mathrm{QE}$ is resonant with the second harmonic of the incident frequency, the QE plays the role of an optical resonator, which efficiently couples to the nonlinear near fields induced around the nanoparticle, extracting them to the far field and thus producing SHG. ${ }^{32}$ This MNP-QE system thus enhances the frequency conversion and allows for its control. To calculate the nonlinear response of the coupled system and to reveal the physical mechanisms behind the SHG in this situation, we use a quantum approach based on the time-dependent density functional theory (TDDFT) ${ }^{45 / 46}$ With the insights obtained from the TDDFT calculation, we develop a semiclassical model. We show that, for the cases where the quantum calculations are doable, the semiclassical model reproduces the TDDFT results. This semiclassical model also allows for addressing more general and complex situations beyond the reach of TDDFT, so that it makes possible a detailed study of the sensitivity of SHG to different parameters that characterize the system. In particular, we demonstrate the polarization conversion of the nonlinear signal, as well as the existence of various regimes of SHG determined by the intrinsic losses of the QE. The methodology and results obtained in our study can pave the conceptual road for enhancing and optimizing second-harmonic generation mediated by quantum emitters coupled to plasmonic systems. $\stackrel{47 / 48}{ }$

\section{TDDFT Calculations}

\section{Nonlinear Response of an Individ- ual Metallic Nanoantenna}

Prior to the discussion of the MNP-QE system, we analyze the nonlinear optical response of an individual spherical MNP calculated within TDDFT. We describe the electronic structure of the MNP within the Jellium model, which is well adapted to TDDFT studies and correctly addresses the quantum many-body dynamics of conduction electrons including the response to strong optical fields. $\underline{49-53}$ The ions at the metal lattice sites are represented by a homogeneous positive background charge of density $n_{+}=\left(\frac{4}{3} \pi r_{s}^{3}\right)^{-1}$ which neutralizes the total charge of the MNP. Atomic units $\mathrm{au}$ are used throughout this paper unless otherwise stated. For the Wigner-Seitz radius, $r_{s}$, we use the typical value for Na metal $r_{s}=4 \mathrm{a}_{0}\left(\mathrm{a}_{0}=0.053 \mathrm{~nm}\right.$ is the Bohr radius). We address a relatively large spherical nanoparticle that contains 1074 conduction electrons, with a radius $a=40.96 \mathrm{a}_{0}(\approx 2.2 \mathrm{~nm})$. The linear optical response of the $\mathrm{Na}$ nanosphere is characterized by the well-developed dipolar plasmon resonance at frequency $\omega_{\mathrm{DP}}=3.17 \mathrm{eV}$, close to that of gold nanoparticles and within the range of excitation energies in organic dyes. ${ }^{54}$ Despite the simplicity of the model, it has successfully offered semiquantitative insights as well as robustly described strong nonlinear effects in plasmonics. $.55-60$ The purpose of the present work is indeed to use a simple, yet realistic modeling scheme to identify and control the main processes governing SHG from a QE coupled to a MNP.

We first perform density functional theory ${ }^{61}$ (DFT) calculations of the ground state of the metallic nanoparticle characterized by an electron density $n_{0}(\mathbf{r})=$ $\sum_{j=\mathrm{occ}} \chi_{j}\left|\Psi_{j}^{0}(\mathbf{r})\right|^{2}$, where the sum runs over the occupied Kohn-Sham (KS) orbitals $\Psi_{j}^{0}(\mathbf{r})$, and the statistical factors $\chi_{j}$ account for both spin and symmetry degeneracy. We then use TDDFT to retrieve the time evolution of the electron density $n(\mathbf{r}, t)=\sum_{j=\mathrm{occ}} \chi_{j}\left|\Psi_{j}(\mathbf{r}, t)\right|^{2}$, when the system is subjected to a quasi-monochromatic external pulse. The time-dependent KS orbitals $\Psi_{j}(\mathbf{r}, t)$ are represented on a spatial mesh in spherical coordinates and their time evolution is obtained from the time-dependent $\mathrm{KS}$ equations ${ }^{45 / 46}$

$$
i \frac{\partial}{\partial t} \Psi_{j}(\mathbf{r}, t)=\hat{H}[n](\mathbf{r}, t) \Psi_{j}(\mathbf{r}, t),
$$

with the use of a short-time propagation algorithm. 62 The initial conditions are given by the ground state of the system: $\Psi_{j}(\mathbf{r}, t=0)=\Psi_{j}^{0}(\mathbf{r})$. The effective Hamiltonian of the system, $\hat{H}[n](\mathbf{r}, t)$, comprises several terms

$$
\hat{H}[n](\mathbf{r}, t)=\underbrace{\hat{T}+V_{\mathrm{H}}[n](\mathbf{r}, t)+V_{\mathrm{xc}}[n](\mathbf{r}, t)}_{\hat{H}_{\mathrm{MNP}}[n](\mathbf{r}, t)}+V_{\mathrm{ext}}(\mathbf{r}, t) \text {. }
$$

In eq. $2 \hat{T}$ is the kinetic energy operator. The Hartree potential, $V_{\mathrm{H}}[n](\mathbf{r}, t)$, is calculated by solving Poisson's equation, $\nabla^{2} V_{\mathrm{H}}[n](\mathbf{r}, t)=-4 \pi\left(n(\mathbf{r}, t)-n_{+}\right)$, where we neglect retardation effects due to the small size of the MNP. The exchange-correlation potential, $V_{\mathrm{xc}}[n](\mathbf{r}, t)$, is described within the adiabatic local density approximation ${ }^{45 / 46}$ (ALDA) using the exchange-correlation kernel of Gunnarsson and Lundqvist. ${ }^{63}$ Finally, $V_{\text {ext }}(\mathbf{r}, t)=$ $\mathbf{r E}_{\text {ext }}(t)$ is the potential of an electron interacting with an incident laser pulse described within the dipole approximation. We consider an incident laser pulse with Gaussian envelope and an electric field given by

$$
\mathbf{E}_{\text {ext }}(t)=\mathrm{E}_{0} \hat{\mathbf{z}} \cos \left(\Omega\left(t-t_{0}\right)\right) e^{-\left(\frac{t-t_{0}}{\sigma}\right)^{2}},
$$

where $\hat{\mathbf{z}}$ is the unit vector along the positive direction of the $z$-axis. The excitation frequency $\Omega$ lies within the 
(a)

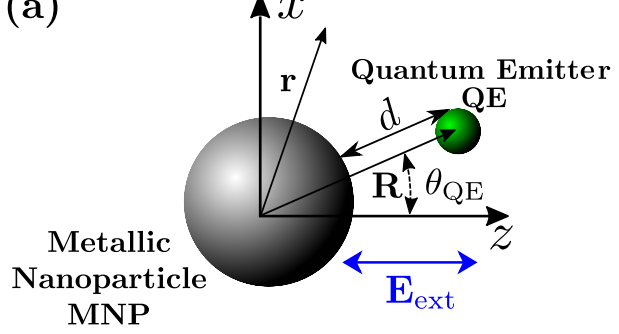

(b)
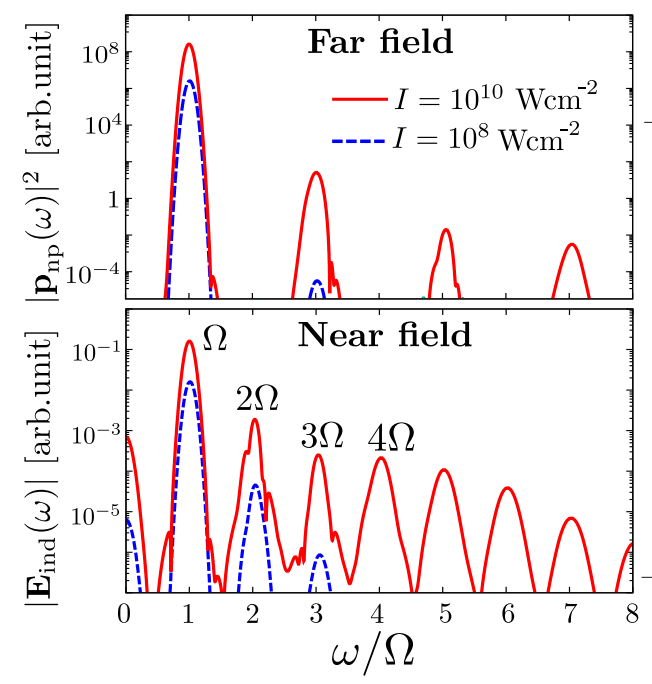

(c)

Individual MNP

Induced Density
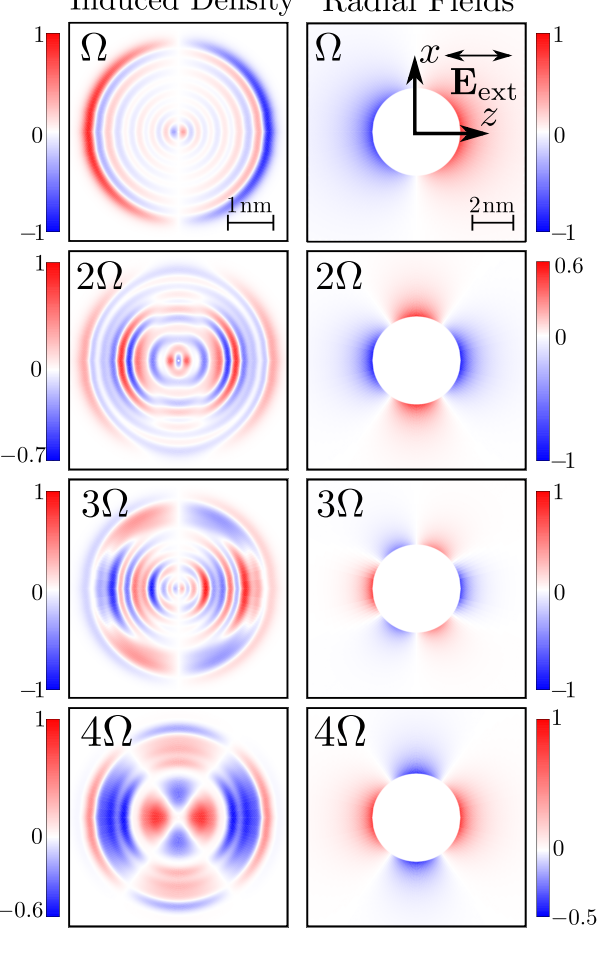

Figure 1: (a) Sketch of the system under study: the radius of the spherical Na nanoparticle is $a=40.96 \mathrm{a}_{0}(2.2 \mathrm{~nm})$, and the point-like QE is located at position $\mathbf{R}$, at a distance $d$ from the nanoparticle surface. (b) Nonlinear optical response of the spherical MNP calculated within TDDFT in the absence of the QE. Results are obtained for an incident $z$-polarized Gaussian electromagnetic pulse with $\Omega=1.585 \mathrm{eV}$ (half of the frequency of the MNP dipolar plasmon $\omega_{\mathrm{DP}}=3.17 \mathrm{eV}$ ), and intensity $I_{0}=10^{8} \mathrm{~W} \mathrm{~cm}^{-2}$ (dashed blue line) and $I_{0}=10^{10} \mathrm{~W} \mathrm{~cm}^{-2}$ (red line). The top panel shows the square of the induced dipole moment $\left|\mathbf{p}_{\mathrm{np}}(\omega)\right|^{2}$, and the bottom panel shows the absolute value of the spectrum of the electric near field $\left|\mathbf{E}_{\text {ind }}(\omega)\right|$ induced at the $z$-axis at $18 \mathrm{a}_{0}(1 \mathrm{~nm})$ from the MNP surface. (c) Color maps of the real part of the charge density (left) and of the radial component of the electric near field (right) induced at the fundamental, second, third and fourth-harmonic frequency by a z-polarized Gaussian electromagnetic pulse with fundamental frequency $\Omega=1.585 \mathrm{eV}$ and intensity $I_{0}=10^{10} \mathrm{~W} \mathrm{~cm}^{-2}$ incident at the individual spherical MNP in the absence of the QE. Results are rotationally symmetric with respect to the $z$-axis, and they are shown in the $(x, z)$-plane normalized to the unity. The coordinates axes are indicated in panel $\mathrm{a}$, and the coordinate origin is at the center of the nanoparticle.

infrared-visible range, and the duration of the pulse is set to $\sigma=5 \times 2 \pi / \Omega$. In our calculations, the arrival time of the pulse $t_{0}$ is set to $t_{0}=5 \sigma$.

In order to analyze the nonlinear optical response of the individual MNP, we calculate the time evolution of the electron density and obtain the timedependent electric near field (induced field) created by the MNP in response to an incident electromagnetic pulse, $\mathbf{E}_{\text {ind }}(\mathbf{r}, t)=\nabla V_{\mathrm{H}}[n(\mathbf{r}, t)]$. We also obtain the induced dipole moment by integrating the differential charge density $\delta n(\mathbf{r}, t)$ over the entire nanoparticle volume $V$, as $\mathbf{p}_{\mathrm{np}}(t)=-\int_{V} \delta n(\mathbf{r}, t) \mathbf{r} d V$, where $\delta n(\mathbf{r}, t)=n(\mathbf{r}, t)-n_{0}(\mathbf{r})$. The frequency-resolved quantities are obtained from the time-to-frequency Fourier transform,

$$
\begin{aligned}
\delta n(\mathbf{r}, \omega) & =\int d t \delta n(\mathbf{r}, t) e^{i \omega t} e^{-\left(\frac{t-t_{0}}{\sigma}\right)^{2}}, \\
\mathbf{p}_{\mathrm{np}}(\omega) & =\int d t \mathbf{p}_{\mathrm{np}}(t) e^{i \omega t} e^{-\left(\frac{t-t_{0}}{\sigma}\right)^{2}}, \\
\mathbf{E}_{\mathrm{ind}}(\mathbf{r}, \omega) & =\int d t \mathbf{E}_{\mathrm{ind}}(\mathbf{r}, t) e^{i \omega t} e^{-\left(\frac{t-t_{0}}{\sigma}\right)^{2}} .
\end{aligned}
$$

The Gaussian filter introduced in eq 4 partially accounts for decay and dephasing processes of the collective density oscillations that are not included in the present (ALDA) TDDFT approach, 64 such as the electron-phonon scattering and the inelastic many-body electron-electron scattering. Employing a Gaussian filter allows one to reach convergent spectral response at high-harmonic frequencies. This approach is justified since the fundamental frequency is strongly detuned 
from the MNP dipolar plasmon resonance so that no electron-density oscillation and high-harmonic generation is expected when the laser is switched off. To be consistent, we apply a Gaussian filter given by the envelope of the incident pulse.

The nonlinear optical response of the spherical MNP calculated with TDDFT in the absence of the QE is analyzed in Figure $1 \mathrm{p}$. In the upper panel, we show the intensity spectrum of the induced dipole moment $\left|\mathbf{p}_{\mathrm{np}}(\omega)\right|^{2}$, which is proportional to the power of the light emitted to the far field. In the lower panel, we show the spectrum of the induced near field $\left|\mathbf{E}_{\text {ind }}(\omega)\right|$ at the $z$-axis, at $18 \mathrm{a}_{0}(\approx 1 \mathrm{~nm})$ from the MNP surface. Results are obtained for an incident Gaussian electromagnetic pulse with fundamental frequency $\Omega=1.585 \mathrm{eV}$ (half of the frequency of the MNP dipolar plasmon $\left.\omega_{\mathrm{DP}}=3.17 \mathrm{eV}\right)$, and intensity $I_{0}=10^{8} \mathrm{~W} \mathrm{~cm}^{-2}\left(\mathrm{E}_{0}=\right.$ $4.8 \times 10^{-5} \mathrm{au}$, dashed blue line) and $I_{0}=10^{10} \mathrm{~W} \mathrm{~cm}^{-2}$ $\left(E_{0}=4.8 \times 10^{-4} \mathrm{au}\right.$, red line), averaged over the duration of the pulse $\sigma$. The corresponding energy per incident pulse is well below the documented damage threshold of small metal nanoparticles.67/69 The induced dipole moment $\left|\mathbf{p}_{\mathrm{np}}(\omega)\right|^{2}$ (upper panel of Figure $1 p$ ) exhibits only odd harmonics. Thus, only odd multiples of the incoming frequency are emitted into the far field consistent with the inversion symmetry of the MNP which prevents even-harmonic generation. $70 / 71$

In contrast to the far-field response, both odd and even harmonics are present in the spectrum of the electric near field induced by the individual MNP (lower panel of Figure 1 $1 \mathrm{p}$ ). Indeed, at the metal-vacuum interface the inversion symmetry is locally broken, and short-range even-harmonic electric fields can be induced close to the metal surface. ${ }^{72 \cdot[6}$ Overall, the nonlinear response for $I_{0}=10^{10} \mathrm{~W} \mathrm{~cm}^{-2}$ is several orders of magnitude larger than that for $I_{0}=10^{8} \mathrm{~W} \mathrm{~cm}^{-2}$. This large increase is in accordance with the $I_{0}^{k / 2}$ dependence of the $k$ th harmonic of the electric fields, expected from the standard theory of nonlinear optics. .70

The color maps of the charge density and of the radial component of the electric near field induced by the incident $z$-polarized Gaussian electromagnetic pulse are shown in Figure 1 k for the fundamental, second, third, and fourth harmonics. The induced charge density $\delta n(\mathbf{r}, \omega=k \Omega)$ of the $k$-th harmonic and the corresponding near field $\mathbf{E}_{\text {ind }}(\mathbf{r}, \omega=k \Omega)$ are shown in the $(x, z)$ plane. Note that because of the symmetry of the configuration, the calculated color maps are independent of a rotation around the $z$-axis. At odd harmonics $(k=1,3)$, the induced densities are antisymmetric with respect to the $(x, y)$-plane, $n(x, y, z, k \Omega)=-n(x, y,-z, k \Omega)$, which results in a net dipole moment (see Figure 1 1 ). In contrast, a quadrupolar-like near field and symmetric charge-density, $n(\mathbf{r}, k \Omega)=n(-\mathbf{r}, k \Omega)$, are induced at even harmonics $(k=2,4) .1977-82$ The dipole moment is zero in this case, and only a weak multipole emission into the far field is possible. Thus, despite the second harmonic being at resonance with the dipolar plasmon of the MNP, the latter can not be excited because of the symmetry selection rules. Another consequence of the symmetry selection rules is that, in the $(x, y)$-plane of symmetry, the even-harmonic near field is oriented perpendicularly to the $z$-polarized incident pulse.

\section{Hybrid Metal Nanoparticle-Quantum Emitter System}

We consider a $\mathrm{QE}$ located in the proximity of the MNP surface as a transducer between the local evenharmonic near field created by the individual nanoparticle and the far-field radiation. The transition frequency of the QE is set such that it is resonant with the second harmonic of the fundamental field. The QE plays the role of an optical resonator, sensitive to the secondharmonic electric near field. ${ }^{[3]}$ We model the QE as a point-like dipole that represents a structureless twolevel system subjected to weak illumination. ${ }^{83 / 84}$ This two-level system represents a HOMO-LUMO transition in a molecule or any electronic transition in an atom or quantum dot. The expectation value of the $\mathrm{QE}$ dipole moment, $\mathbf{p}_{\mathrm{QE}}(t)$, evolves in time according to 85

$$
\ddot{\mathbf{p}}_{\mathrm{QE}}(t)+\gamma_{\mathrm{QE}} \dot{\mathbf{p}}_{\mathrm{QE}}(t)+\omega_{0}^{2} \mathbf{p}_{\mathrm{QE}}(t)=\alpha_{0} \mathbf{E}_{\mathrm{tot}}(\mathbf{R}, t),
$$

where $\gamma_{\mathrm{QE}}$ refers to the intrinsic losses of the $\mathrm{QE}, \alpha_{0}$ is the $\mathrm{QE}$ oscillator strength, and $\omega_{0}$ is the $\mathrm{QE}$ resonant frequency. As we already stated earlier, we are interested in the case when $\omega_{0}=2 \Omega$. Thus, the $\mathrm{QE}$ is set to be resonant with the second-harmonic frequency in all our calculations, so that a variation of $\Omega$ implies simultaneous variation of $\omega_{0}$. For simplicity, $\alpha_{0}$ is always taken as a scalar $\alpha_{0}=1 \mathrm{au}$, corresponding to an isotropic $\mathrm{QE}$. In the TDDFT calculations we consider intrinsic losses $\gamma_{\mathrm{QE}}=0.1 \mathrm{eV}$.

The total electric field $\mathbf{E}_{\text {tot }}(\mathbf{R}, t)$ acting on the $Q E$ is given by the sum of the incident laser field $\mathbf{E}_{\text {ext }}(t)$ and the field $\mathbf{E}_{\text {ind }}(\mathbf{R}, t)$ induced by the MNP at the position of the QE, $\mathbf{E}_{\text {tot }}(\mathbf{R}, t)=\mathbf{E}_{\text {ext }}(t)+\mathbf{E}_{\text {ind }}(\mathbf{R}, t)$. Note that $\mathbf{E}_{\text {ind }}(\mathbf{R}, t)$ includes the reaction of the MNP not only to the incident pulse, but also to the presence of the QE. It is thus responsible for the QE self-interaction. To verify that the transition is not saturated (and thus that the description of the $\mathrm{QE}$ as a classical dipole given by eq 5 holds), we performed additional calculations where we explicitly addressed the quantum dynamics of the two-level system treated in the basis of the ground and excited states.

The QE dipole acts as an additional radiation source emitting into the far field as well as affecting the dynamics of the conduction electrons of the MNP. Because of the small size of the system, retardation effects can be neglected, so that the QE placed at a position $\mathbf{R}$ near the MNP induces an electric potential given by

$$
V_{\mathrm{QE}}(\mathbf{r}, t)=-\mathbf{p}_{\mathrm{QE}}(t) \cdot \frac{\mathbf{r}-\mathbf{R}}{|\mathbf{r}-\mathbf{R}|^{3}},
$$


where the transition charge density of the QE is represented using the point-dipole approximation. Thus, the effective Hamiltonian acting on the conduction electrons of the MNP, which includes the QE potential, becomes: from eq2 into

$$
\hat{H}[n](\mathbf{r}, t)=\hat{H}_{\mathrm{MNP}}[n](\mathbf{r}, t)+V_{\mathrm{ext}}(\mathbf{r}, t)+V_{\mathrm{QE}}(\mathbf{r}, t) .
$$

Equations 1, 5, 6, and 7/are solved self-consistently allowing to obtain the frequency-resolved quantities of interest such as the dipole moment induced in the MNP $\mathbf{p}_{\mathrm{np}}(\omega)$, in the QE $\mathbf{p}_{\mathrm{QE}}(\omega)$, and the total dipole moment given by the sum of both, $\mathbf{p}(\omega)=\mathbf{p}_{\mathrm{np}}(\omega)+\mathbf{p}_{\mathrm{QE}}(\omega)$.

We initially place the QE at the $z$-axis, corresponding to the direction of polarization of the incident laser pulse. For this geometry, only z-polarized dipole moments are induced in the QE and in the MNP. The system then possesses cylindrical symmetry with respect to the $z$-axis, which greatly reduces the computational demands.

We use an incident $z$-polarized Gaussian electromagnetic pulse with intensity $I_{0}=10^{10} \mathrm{~W} \mathrm{~cm}^{-2}$, and fundamental frequency $\Omega=1.585 \mathrm{eV}$ such that the second harmonic is at resonance with the dipolar plasmon of the MNP. The electric field of the pulse is shown with dashed black line in Figure $2 \mathrm{a}$. The QE is located at $d=18 \mathrm{a}_{0}(\approx 1 \mathrm{~nm})$ from the MNP surface. A dipole moment $\mathbf{p}_{\mathrm{OE}}(t)$ induced at the $\mathrm{QE}$ due to its interaction with the MNP is shown in Figure 2 by the red line. The resonant excitation of the QE manifests itself at long evolution times after the passage of the electromagnetic pulse where $\mathbf{p}_{\mathrm{QE}}(t)$ oscillates at the $\mathrm{QE}$ transition frequency, $\omega_{0}=2 \Omega$. The excited $Q E$ strongly modifies the nonlinear response of the system.

In contrast with the individual MNP, the hybrid MNPQE structure features strong emission at the even harmonics, as we show in Figure 2 $\mathrm{p}$. In this figure the intensity spectrum of the total induced dipole moment $|\mathbf{p}(\omega)|^{2}$ of the hybrid MNP-QE structure is shown by the blue line. The reference results obtained for the nonlinear response of the individual MNP (without QE) are shown by the dashed red line. The even harmonics in the far field emerge because the QE breaks the reflection symmetry with respect to the $(x, y)$-plane, and thus the total inversion symmetry of the system. ${ }^{18}$ Note that the spectra in Figure $2 \mathrm{p}$ are artificially broadened because of the Gaussian filter (see eq 4). In the next section we introduce a semiclassical method that allows to overcome the difficulty of the (ALDA) TDDFT calculations to describe relaxation processes induced by the interaction of excited electrons with phonons and by many-body inelastic scattering events. 64 , 66

The resonance between the transition frequency of the QE and the second harmonic of the incident light greatly enhances the intensity emitted by the system at $2 \Omega$. To illustrate this resonance effect, in the inset of Figure $2 p$, we show the results obtained for a different situation. The QE transition is set resonant with $4 \Omega$, and the system is illuminated by a Gaussian pulse with fundamental frequency $\Omega$ such that the fourth harmonic matches the frequency of the MNP dipolar plasmon, $4 \Omega=\omega_{\mathrm{DP}}=3.17 \mathrm{eV}$. For this excitation frequency, $|\mathbf{p}(\omega)|^{2}$ at the second harmonic decreases by several orders of magnitude in favor of the emission at the fourth harmonic resonant with the QE transition.

Let us now focus on the physical mechanism determining the nonlinear second-harmonic response of the hybrid MNP-QE system. The polarization of the QE mainly oscillates at the fundamental and at the secondharmonic frequency of the incident pulse. Indeed, the QE transition is resonant with the second harmonic, however the field acting on the QE at the fundamental frequency is orders of magnitude stronger than the near field induced by the MNP at $2 \Omega$. As a result, the offresonant polarization of the QE might be strong. Thus, two distinct channels might contribute to the secondharmonic polarization of the coupled MNP-QE system: (i) the excitation of the QE at the resonant secondharmonic frequency and associated QE polarization induces a second-harmonic dipole moment in the MNP due to the linear interaction, and (ii) the off-resonant polarization of the $\mathrm{QE}$ at the fundamental frequency lifts the symmetry constraint as far as the entire system is considered and allows for the second-harmonic dipole moment to be induced via the nonlinear process.

In order to obtain the respective weight of the different channels inducing a second-harmonic dipole at the MNP, we proceed as follows. Using the Fourier analysis, we split the dipole moment of the QE obtained from the TDDFT calculation into a sum of the two leading contributions, $\mathbf{p}_{\mathrm{QE}}(t)=\mathbf{p}_{\mathrm{QE}}^{\Omega}(t)+\mathbf{p}_{\mathrm{QE}}^{2 \Omega}(t)$, one oscillating at the fundamental frequency, $\mathbf{p}_{\mathrm{QE}}^{\Omega}(t)$, and the other one at the second-harmonic frequency, $\mathbf{p}_{\mathrm{QE}}^{2 \Omega}(t)$. We then run two different TDDFT simulations, where the electron density of the MNP evolves (i) solely under the action of the potential created by the point dipole oscillating at the second-harmonic frequency, $V_{\mathrm{QE}}(\mathbf{r}, t)=-\mathbf{p}_{\mathrm{QE}}^{2 \Omega}(t) \cdot \frac{\mathbf{r}-\mathbf{R}}{|\mathbf{r}-\mathbf{R}|^{3}}$, with no external laser pulse applied $\left(V_{\text {ext }}=0\right)$, and (ii) under the action of the external laser pulse and the potential created by the point dipole oscillating at the fundamental frequency, $V_{\mathrm{QE}}(\mathbf{r}, t)=-\mathbf{p}_{\mathrm{QE}}^{\Omega}(t) \cdot \frac{\mathbf{r}-\mathbf{R}}{|\mathbf{r}-\mathbf{R}|^{3}}$. The self-consistency loop is stopped in both cases, in the sense that the MNP does not act back on the QE. More details of this procedure can be found in the Supporting Information.

As shown in Figure 2 , the second-harmonic response of the MNP obtained in scenario $i$ and shown with blue line, closely corresponds to the result of the complete self-consistent calculation (black line). The secondharmonic response obtained under scenario ii is smaller by more than 2 orders of magnitude (red line). These results point toward the following main physical mechanism underlying the far-field SHG in the present system:

- The interaction of the (strong) incident laser pulse with the conduction electrons of the MNP generates a second-harmonic near field. 
(a)
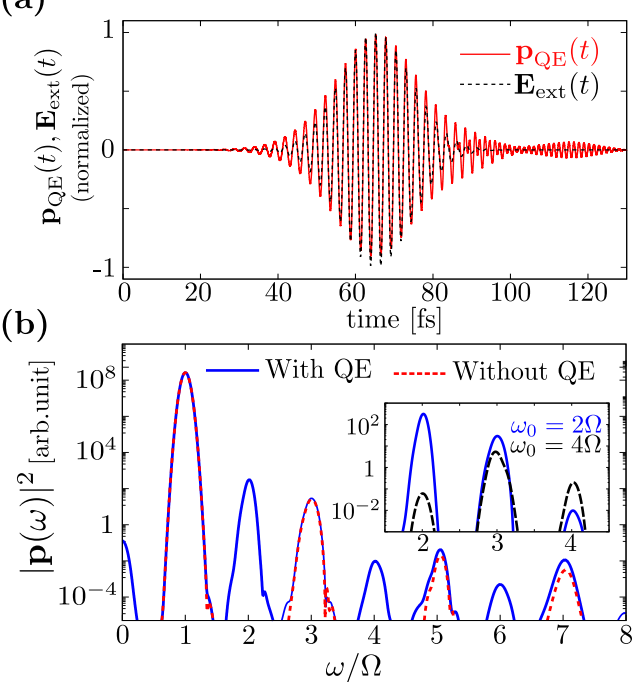

(c)
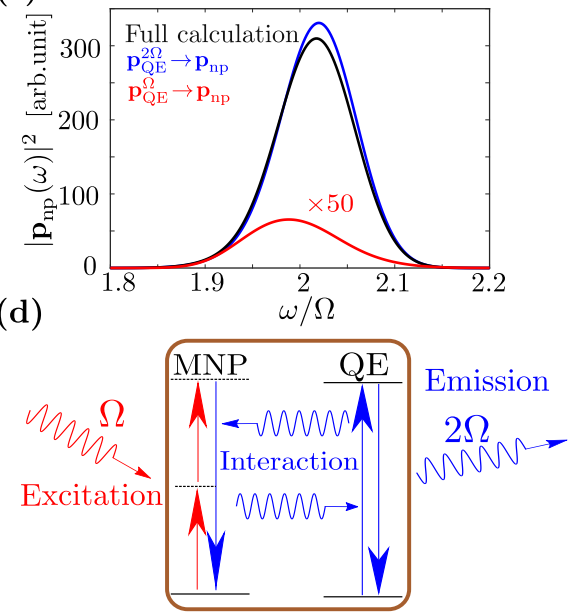

Figure 2: (a) Time evolution of an incident $z$-polarized Gaussian electromagnetic pulse $\mathbf{E}_{\text {ext }}(t)$ with fundamental frequency $\Omega=1.585 \mathrm{eV}$ (dashed black line) and of the dipole moment $\mathbf{p}_{\mathrm{QE}}(t)$ of the QE located at $d=18 \mathrm{a}_{0}(1 \mathrm{~nm})$ from the MNP surface (solid red line). Results are normalized to their maximum value. (b) Intensity spectrum of the total dipole moment $|\mathbf{p}(\omega)|^{2}$ of the hybrid MNP-QE system (solid blue line) and of the individual MNP (dashed red line). Results are obtained for an incident $z$-polarized Gaussian electromagnetic pulse with fundamental frequency $\Omega=1.585 \mathrm{eV}$ and intensity $I_{0}=10^{10} \mathrm{~W} \mathrm{~cm}^{-2}$. In the inset, the solid blue line corresponds to the same result as in the main panel, and the dashed black line corresponds to the results obtained for the transition frequency of the QE resonant with the fourth harmonic of the incident light, $\omega_{0}=4 \Omega$, with $\Omega=0.79 \mathrm{eV}$. (c) Second-harmonic dipole moment of the MNP when interacting with the QE from a full calculation (black line), when interacting with the QE oscillating only at $2 \Omega$ (blue line), and when interacting with the QE oscillating at $\Omega$ (red line, amplified 50 times) for the same parameters as in panel b. For further details see the main text. (d) Sketch of the main physical process behind the SHG in the present system. The MNP induces a near field at $2 \Omega$ that excites the emitter. Then, the linear MNP-QE interaction at $2 \Omega$ generates the emission into the far field at the second-harmonic frequency.

- The second harmonic of the near field of the MNP resonantly drives the dipolar polarization of the $Q E$ at $2 \Omega$.

- The resulting $Q E$ dipole oscillates at $2 \Omega$ and radiates into the far field in the presence of the MNP antenna.

The physical process revealed by the TDDFT simulations (sketched in Figure 2 $\mathrm{d}$ ) is consistent with the mechanism considered in previous related work,, 44 and it gives rise to a practical semiclassical approach that can be used to describe more complex situations due to the intrinsic losses of the QE or its position. We develop this semiclassical approach in the following section.

\section{Semiclassical Model}

The understanding of the physical mechanism behind the SHG established above with the TDDFT can be used to develop a semiclassical model capable of reproducing the role of the QE in the second-harmonic response of the coupled system. This model goes beyond the TDDFT because it naturally incorporates the losses and the plasmon decay, and thus lifts the necessity of using artificial broadening of the spectral features. Moreover, it allows one to analyze systems without axial symmetry, i.e., situations of arbitrary polarization of the incident pulse and location of the QE. An analysis of the latter would be computationally out of reach for the TDDFT calculations.

In this semiclassical model, the QE is excited by the incident laser pulse and by the nonlinear electric near field induced by the MNP. This nonlinear near field is obtained from the TDDFT calculations of the individual MNP, i.e., in the absence of the QE. The excited QE then radiates into the far field in the presence of the MNP. The last stage of the calculation, which involves the coupling of the QE with the MNP and the resulting light emission to the far field, is treated within a classical framework. Using the dyadic Green's function formalism, $\underline{84}$ we obtain

$$
\begin{aligned}
& \mathbf{p}_{\mathrm{QE}}(\omega)= \\
& \quad\left(\mathbb{I}-\alpha_{\mathrm{QE}}(\omega) \stackrel{\leftrightarrow}{G}(\mathbf{R}, \mathbf{R}, \omega)\right)^{-1} \alpha_{\mathrm{QE}}(\omega) \mathbf{E}_{\mathrm{tot}}(\mathbf{R}, \omega), \\
& \mathbf{p}_{\mathrm{np}}(\omega)=\mathbf{p}_{\mathrm{np}}^{\mathrm{TDDFT}}(\omega)+\alpha_{\mathrm{np}}(\omega) \stackrel{\leftrightarrow}{G}_{0} \mathbf{p}_{\mathrm{QE}}(\omega),
\end{aligned}
$$

where $\mathbf{p}_{\mathrm{np}}^{\mathrm{TDDFT}}(\omega)$ is the dipole moment of the individual MNP induced by the incident laser pulse, calculated 
with TDDFT in the absence of the QE. This contribution is zero for even harmonics. The electric field at the position $\mathbf{R}$ of the $\mathrm{QE}, \mathbf{E}_{\mathrm{tot}}(\mathbf{R}, \omega)$, is given by

$$
\mathbf{E}_{\text {tot }}(\mathbf{R}, \omega)=\mathbf{E}_{\text {ind }}^{\mathrm{TDDFT}}(\mathbf{R}, \omega)+\mathbf{E}_{\mathrm{ext}}(\omega),
$$

where the field of the external laser pulse $\mathbf{E}_{\text {ext }}(\omega)$ is added to the electric field $\mathbf{E}_{\text {ind }}^{\mathrm{TDDFT}}(\mathbf{R}, \omega)$ created by the noninteracting MNP. The field $\mathbf{E}_{\text {ind }}^{\mathrm{TDDFT}}(\mathbf{R}, \omega)$ is obtained from the TDDFT calculation for the individual MNP subjected to a Gaussian laser pulse, and it introduces all the second-harmonic nonlinearity. The polarizability of the $\mathrm{QE}, \alpha_{\mathrm{QE}}(\omega)$, and that of the Na nanoparticle of radius $a, \alpha_{\mathrm{np}}(\omega)$, are found from

$$
\begin{aligned}
\alpha_{\mathrm{QE}}(\omega) & =\frac{\alpha_{0}}{\left(\omega_{0}^{2}-\omega^{2}-i \omega \gamma_{\mathrm{QE}}\right)} \\
\alpha_{\mathrm{np}}(\omega) & =a^{3} \frac{\epsilon_{m}(\omega)-1}{\epsilon_{m}(\omega)+2}
\end{aligned}
$$

where

$$
\epsilon_{m}(\omega)=1-\frac{\omega_{p}^{2}}{\omega^{2}+i \gamma \omega}
$$

stands for the metal dielectric function described within the Drude model, with plasma frequency $\omega_{p}$ and intrinsic loss rate $\gamma$.

Finally, in eq $8 \overleftrightarrow{G}_{0}$ and $\overleftrightarrow{G}(\mathbf{R}, \mathbf{R}, \omega)$ are the dyadic Green's functions: $\stackrel{\leftrightarrow}{G}_{0}$ provides the electric field at the center of the MNP produced by a unitary point dipole placed at position $\mathbf{R} \cdot \sqrt{85} \stackrel{\leftrightarrow}{G}(\mathbf{R}, \mathbf{R}, \omega)$ is the self-interaction dyadic Green's function that expresses the electric field created by the MNP at position $\mathbf{R}$ in response to a point dipole located at the same position. This can be fully defined by the components perpendicular and parallel to the MNP surface, 88

$$
\begin{aligned}
G_{\perp}(\mathbf{R}, \mathbf{R}, \omega) & =\sum_{l=1}^{l_{\max }} \frac{\epsilon_{m}(\omega)-1}{\epsilon_{m}(\omega)+\frac{l+1}{l}} \frac{a^{2 l+1}}{|\mathbf{R}|^{2 l+4}}(l+1)^{2}, \\
G_{\|}(\mathbf{R}, \mathbf{R}, \omega) & =\sum_{l=1}^{l_{\max }} \frac{\epsilon_{m}(\omega)-1}{\epsilon_{m}(\omega)+\frac{l+1}{l}} \frac{a^{2 l+1}}{|\mathbf{R}|^{2 l+4}} \frac{1}{2} l(l+1),
\end{aligned}
$$

where the summation over $l$ represents a multipole expansion with a formally infinite number of multipoles $\left(l_{\max }=\infty\right)$. In practice, we found that $l_{\max } \sim 15$ guarantees the convergence of the Green's function for our system. The metal dielectric function $\epsilon_{m}(\omega)$ is given by eq 11. where the plasma frequency $\omega_{p}=5.49 \mathrm{eV}$ and attenuation $\gamma=0.218 \mathrm{eV}$ are determined from the fit to the plasmon resonance in the optical absorption spectrum of the individual MNP calculated with linearresponse TDDFT. These parameters, fixed prior to the Green's function calculation, are used throughout our work for the Drude description of the metal nanoparticle in the semiclassical model. This parametrization allows to account for the finite-size effects on the localized plasmon energy and Landau damping in the MNP. ${ }^{4989}$ For further details we address the reader to the Supporting Information.

As reported in the literature,, 0019 the induced charges are generally not located exactly at the geometrical surface, but they are instead shifted inward or outward, depending on the actual band structure of the material. Therefore, to account for this effect, we introduce a distance shift $\Delta d$ in the semiclassical model. In practice, for the QE located at a distance $d$ from the geometrical surface of the MNP the semiclassical model calculations are performed for a reduced "effective" separation $d_{\text {eff }}=d-\Delta d$, where $\Delta d=1.5 \mathrm{a}_{0}$. The TDDFT results naturally include the spill-out effect and are not a subject to the distance shift. As a result, e.g., the TDDFT data obtained for the QE located at $d=18 \mathrm{a}_{0}$ from the MNP surface has to be compared with semiclassical model results obtained for $d_{\text {eff }}=16.5 \mathrm{a}_{0}$.

\section{Results and Discussion}

\section{TDDFT vs Semiclassical Model Results}

A detailed comparison between the TDDFT and semiclassical model calculations is shown in Figure 3 Along with the overall good agreement, which establishes the validity of the semiclassical model, the results of the quantum and semiclassical approaches reveal some differences. Below we discuss the similarities and differences observed.

In Figure 3 a, we start with the comparison between the Green's function $G_{\perp}(\mathbf{R}, \mathbf{R}, \omega)$ obtained from the classical expression in eq 12 and the corresponding result from the TDDFT calculations. Within the TDDFT, we apply the standard linear-response procedure, where the MNP is excited by an impulsive perturbation given by the potential of a small $z$-oriented dipole $\zeta$ located at $\mathbf{R}=(0,0, a+d), V_{\text {ext }}(\mathbf{r}, t)=-\hat{\mathbf{z}} \cdot \frac{\mathbf{r}-\mathbf{R}}{|\mathbf{r}-\mathbf{R}|^{3}} \zeta \delta(t)$. Here $\delta(t)$ is the Dirac delta function. From the time-dependent electron density $n(\mathbf{r}, t)$ we obtain the $z$-component of the time-dependent field induced at the dipole position $\mathcal{F}(\mathbf{R}, t)$. Finally, the time-to-frequency Fourier transform yields the frequency-resolved quantity $\frac{1}{\zeta} \mathcal{F}(\mathbf{R}, \omega)$, which can be directly compared with classical result $G_{\perp}(\mathbf{R}, \mathbf{R}, \omega)$.

In general, in Figure $3 \mathrm{a}$, both the semiclassical model (dashed lines) and quantum (solid lines) results show good agreement in the range $\omega=2-3.4 \mathrm{eV}$, with a dipolar plasmon resonance of similar strength at $\omega_{\text {DP }}=3.17 \mathrm{eV}$. However, two clear differences between the two approaches can be observed: First, for the quantum description of the MNP, along with the collective plasmon features, intense single particle electron-hole pair excitations appear at low energies $(\omega \sim 1-2 \mathrm{eV})$ because of the finite size of the system. This quantum 
(a)

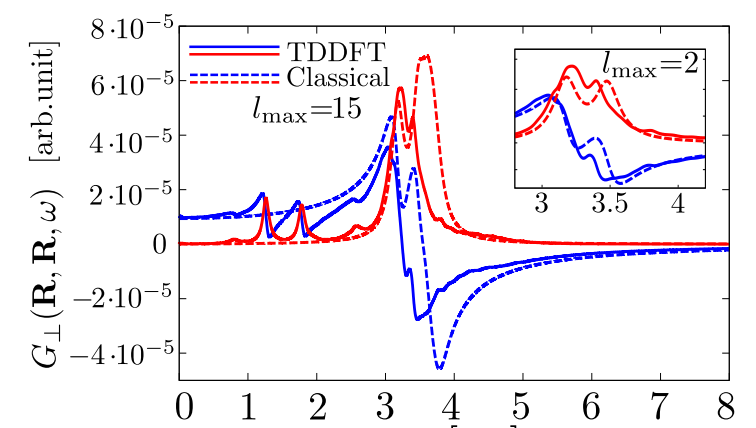

(b)

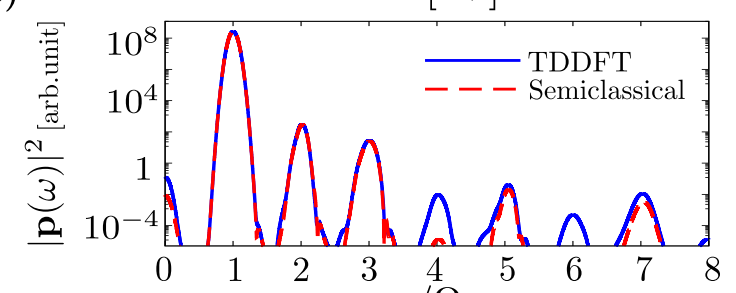

(c)

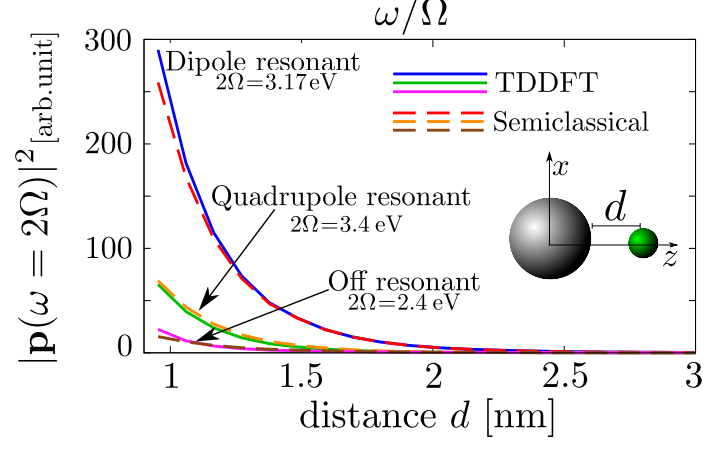

Figure 3: Comparison between the TDDFT and semiclassical model results for the QE placed at the z-axis in front of the MNP. (a) Real (blue) and imaginary (red) part of the frequency-dependent self-interaction dyadic, $G_{\perp}(\mathbf{R}, \mathbf{R}, \omega)$, for a $z$-polarized point dipole located at distance $d=26 \mathrm{a}_{0}(1.3 \mathrm{~nm})$ from the MNP surface. Dashed lines: eq 12 with $l_{\max }=15$. Solid lines: TDDFT. Results obtained with $l_{\max }=2$ (i.e., including only the two lowest-order collective excitations of the MNP) are compared with the TDDFT data in the inset. (b) Intensity spectrum of the induced total dipole $|\mathbf{p}(\omega)|^{2}$ obtained from the TDDFT calculations (blue line) and from the semiclassical model (red line). Results are shown as a function of the frequency measured in units of the fundamental frequency $\Omega=1.585 \mathrm{eV}$ of the incident laser pulse. The QE is located at $d=18 \mathrm{a}_{0}$ $(1 \mathrm{~nm})$ from the MNP surface. (c) Second-harmonic dipole $|\mathbf{p}(\omega=2 \Omega)|^{2}$ calculated using the semiclassical model (dashed lines) and TDDFT (solid lines). Results are shown as a function of the MNP-QE distance $d$ for three different fundamental frequencies $2 \Omega=2.4 \mathrm{eV}$, $2 \Omega=3.17 \mathrm{eV}$, and $2 \Omega=3.4 \mathrm{eV}$. In panels $\mathrm{b}$ and $\mathrm{c} \mathrm{a} z-$ polarized Gaussian pulse of intensity $I_{0}=10^{10} \mathrm{~W} \mathrm{~cm}^{-2}$ is used.

effect is absent in the semiclassical model based on the Drude dielectric function of the free-electron gas. As a second difference, the higher-order multipole plas- monic resonances at $\omega \sim 3.5 \mathrm{eV}$ are less developed in the quantum response as compared to the semiclassical one. This is connected to the diffused nature of the surface-charge density in the quantum description due to dynamical nonlocal screening, which does not allow higher-order pasmon modes to be formed in small nanoparticles. A larger MNP would be needed to obtain well-defined multipolar plasmon modes. To illustrate the effect of the multipolar excitations, we performed an additional calculation of $G_{\perp}(\mathbf{R}, \mathbf{R}, \omega)$ by only considering the lowest dipolar and quadrupolar terms $\left(l_{\max }=2\right)$ in the multipole expansion given by eq 12 . Much better agreement with the TDDFT results is obtained in this case at energies near $3.5 \mathrm{eV}$ as shown by the inset of Figure 3 a. We checked that the agreement between the TDDFT and the semiclassical model improves when increasing the MNP-QE separation $d$, consistent with the decrease of the relative contribution of the highorder multipoles with increasing $d$ (see eq 12 where $|\mathbf{R}|=a+d)$.

We further validate the semiclassical approach by comparing the TDDFT and semiclassical results for the nonlinear response of the hybrid MNP-QE system excited by a $z$-polarized Gaussian pulse with fundamental frequency $\Omega=1.585 \mathrm{eV}$ and intensity $I_{0}=$ $10^{10} \mathrm{~W} \mathrm{~cm}^{-2}$. In Figure $3 \mathrm{~b}$ we show the intensity spectrum of the total dipole $|\mathbf{p}(\omega)|^{2}$ obtained with the TDDFT (solid blue line) and the semiclassical model (dashed red line) calculations. An excellent agreement between the two approaches is obtained for the nonlinear polarization at the second harmonic, which is at the focus of the present work.

For higher-order harmonics, in particular the higherorder even harmonics that can be generated only owing to the interaction with the $\mathrm{QE}$, the agreement strongly worsens. This indicates that other nonlinear processes beyond the scope of the present model become important for such low intensity emission. It is worth noting that for the sake of comparison between the two approaches, the Gaussian filters employed in the Fourier analysis of the time-dependent quantities in the TDDFT are also accounted for in the semiclassical model calculations shown in panels $b$ and c of Figure 3, as we detail in the Supporting Information.

The results shown so far were obtained for the second harmonic at resonance with the dipolar plasmon of the MNP. One would expect that under these conditions the SHG is very efficient. Indeed, the emission of the QE resonantly excited by the $2 \Omega$ near field of the MNP is further enhanced by the dipolar resonance of the MNP playing the role of a nanoantenna. $\frac{89126-32}{-32}$ On the other hand, the second-harmonic near field of the MNP that drives the QE, $\mathrm{E}_{\text {ind }}^{\mathrm{TDDT}}(\mathbf{R}, \omega=2 \Omega)$, has a quadrupolar character ${ }^{19] 77-82 \mid 92}$ (see Figure 1 F). Therefore, it should be stronger when the second-harmonic frequency matches the quadrupolar plasmon resonance of the MNP ${ }^{[92}\left(2 \Omega=\omega_{\mathrm{QP}}=3.4 \mathrm{eV}\right.$, see Supporting Information). This, in turn, should also lead to an efficient excitation of the QE and thus increase the emitted 
second-harmonic signal.

In order to find the optimal conditions for the SHG, we use the TDDFT and the semiclassical method to study how the second-harmonic radiation into the far field depends on the fundamental frequency. In Figure 3f, the intensity spectrum $|\mathbf{p}(\omega=2 \Omega)|^{2}$ is shown as a function of the distance $d$ of the QE from the MNP surface for three different frequencies $\Omega$ of the incident Gaussian laser pulse. First, the values of $\Omega$ are set such that the second harmonic matches the dipolar $(2 \Omega=3.17 \mathrm{eV}$, dipole resonant) and the quadrupolar $(2 \Omega=3.4 \mathrm{eV}$, quadrupole resonant) plasmonic resonances of the MNP. We consider as well the reference case where both the fundamental frequency and the second harmonic are out of resonance with any of the plasmonic modes of the MNP $(2 \Omega=2.4 \mathrm{eV}$, off resonant). We recall that the transition frequency of the $\mathrm{QE}$ is always set resonant with the second harmonic, $\omega_{0}=2 \Omega$.

As a first observation from the results shown in Figure 3 , we would like to emphasize that the semiclassical model does an excellent job in reproducing the TDDFT results. Both approaches show the strongest SHG when the second-harmonic frequency matches the dipolar plasmon resonance of the MNP, while the off-resonant conditions lead to the smallest SHG. Furthermore, for all three excitation frequencies the power emitted to the far field at the second harmonic monotonically decreases with increasing distance $d$. This behavior reflects the decrease of the nonlinear near field created by the MNP. However, as we show using the semiclassical model in the next section, the frequency and distance dependence of the SHG is strongly dependent on the intrinsic losses of the QE. This aspect of the problem cannot be addressed within the present TDDFT because it lacks the description of the decay and dephasing processes,, 6465 forcing an artificial broadening of the spectral features with, e.g., the Gaussian filters applied to connect the time-resolved and the frequency-resolved quantities. As a consequence, reliable information can only be obtained from TDDFT for the cases when the QE has large intrinsic losses.

\section{Influence of the Losses and Posi- tion of the QE in the SHG}

The semiclassical model established in the previous section can be used for a detailed study of the SHG from the hybrid MNP-QE structure in response to incident plane-wave illumination. In particular, the analysis of the dependence of the SHG on experimentally relevant parameters, which was not computationally possible with TDDFT, becomes within reach. Namely, we are interested in the dependence of the SHG on the fundamental frequency, on the intrinsic QE losses, and on the relative position of the QE.

The relationship between the second-harmonic dipole $\overrightarrow{\mathcal{P}}_{2 \Omega}$ induced by an incident plane wave with average in- tensity $I_{0}$ and previous results of the frequency-resolved dipole $\mathbf{p}(\omega)$ induced by an electromagnetic Gaussian pulse (eq 3 is given by

$$
\overrightarrow{\mathcal{P}}_{2 \Omega}=\frac{8 \sqrt{2 \pi} I_{0}}{\sigma c \mathrm{E}_{0}^{2}} \mathbf{p}(\omega=2 \Omega),
$$

where $c$ is the speed of light in vacuum, and $\sigma$ and $\mathrm{E}_{0}$ are the duration and the amplitude of the electromagnetic Gaussian pulse used in the TDDFT simulations, respectively (see details in the Supporting Information). $\mathbf{p}(\omega=2 \Omega)$ is calculated using the semiclassical model (eq 8$)$.

The color maps in Figure 4 show the second-harmonic response $\left|\overrightarrow{\mathcal{P}}_{2 \Omega}\right|^{2}$ of the coupled MNP-QE structure subjected to an incident $z$-polarized plane wave with frequency $\Omega$ and average intensity $I_{0}=10^{10} \mathrm{~W} \mathrm{~cm}^{-2}$ for different locations of the QE, defined by the distance $d$ from the nanoparticle surface and the polar angle $\theta_{\mathrm{QE}}$ (see insets in Figure 1a and Figure 4 a for the geometry of the system). The range of distances $d=1-5 \mathrm{~nm}$ covered in our study is set such that retardation effects can be neglected. The results are axially symmetric with respect to the $z$-axis, and without loss of generality, we consider that the QE is placed in the $(x, z)$-plane.

Figure 4 shows the dependence of the SHG on the QE position different fundamental frequencies, $\Omega$, and intrinsic losses of the $\mathrm{QE}, \gamma_{\mathrm{OE}}$. Specifically, we performed calculations for (i) high QE losses $\left(\gamma_{\mathrm{QE}}=0.1 \mathrm{eV}\right)$, which often occur in experiments at room temperature, (ii) intermediate losses $\left(\gamma_{\mathrm{QE}}=10^{-3} \mathrm{eV}\right)$, and (iii) low losses $\left(\gamma_{\mathrm{QE}}=10^{-7} \mathrm{eV}\right)$, close to the spontaneous decay rate of the QE. Similar to the discussion in Figure 35, we consider the fundamental frequencies corresponding to three different situations: (i) second harmonic is at resonance with the dipolar plasmon of the MNP (dipole resonant); (ii) second harmonic is at resonance with the quadrupolar plasmon of the MNP (quadrupole resonant); (iii) the second harmonic and the fundamental frequency are off-resonance with any plasmon mode of the MNP (off resonant).

Some common features can be pointed out in Figure 4 First, the symmetry with respect to the position of the emitter given by the angle $\theta_{\mathrm{QE}}=\pi / 2$, which reflects the symmetry of the system with respect to the $(x, y)$ plane. Second, the SHG is generally stronger for the QE placed around the $z$-axis $\left(\theta_{\mathrm{QE}} \approx 0\right.$ and $\left.\pi\right)$, the region where the near field excited at $2 \Omega$ is more intense (due to its quadrupolar spatial profile, see Figure $1 \mathrm{k}$ ). Indeed, the near field resonantly excites the QE and partially imprints its spatial distribution into the dependence of the second-harmonic signal on the position of the QE. As another result common for large and intermediate QE losses: the strongest SHG is obtained for the second-harmonic frequency at resonance with the dipolar plasmon of the metal nanoparticle, which corroborates the TDDFT results discussed in the previous section. The maximum second-order nonlinear hyperpolarizability $\alpha_{\hat{\mathbf{m}}}^{(2)}(2 \Omega ; \Omega, \Omega$ ) (which relates the 

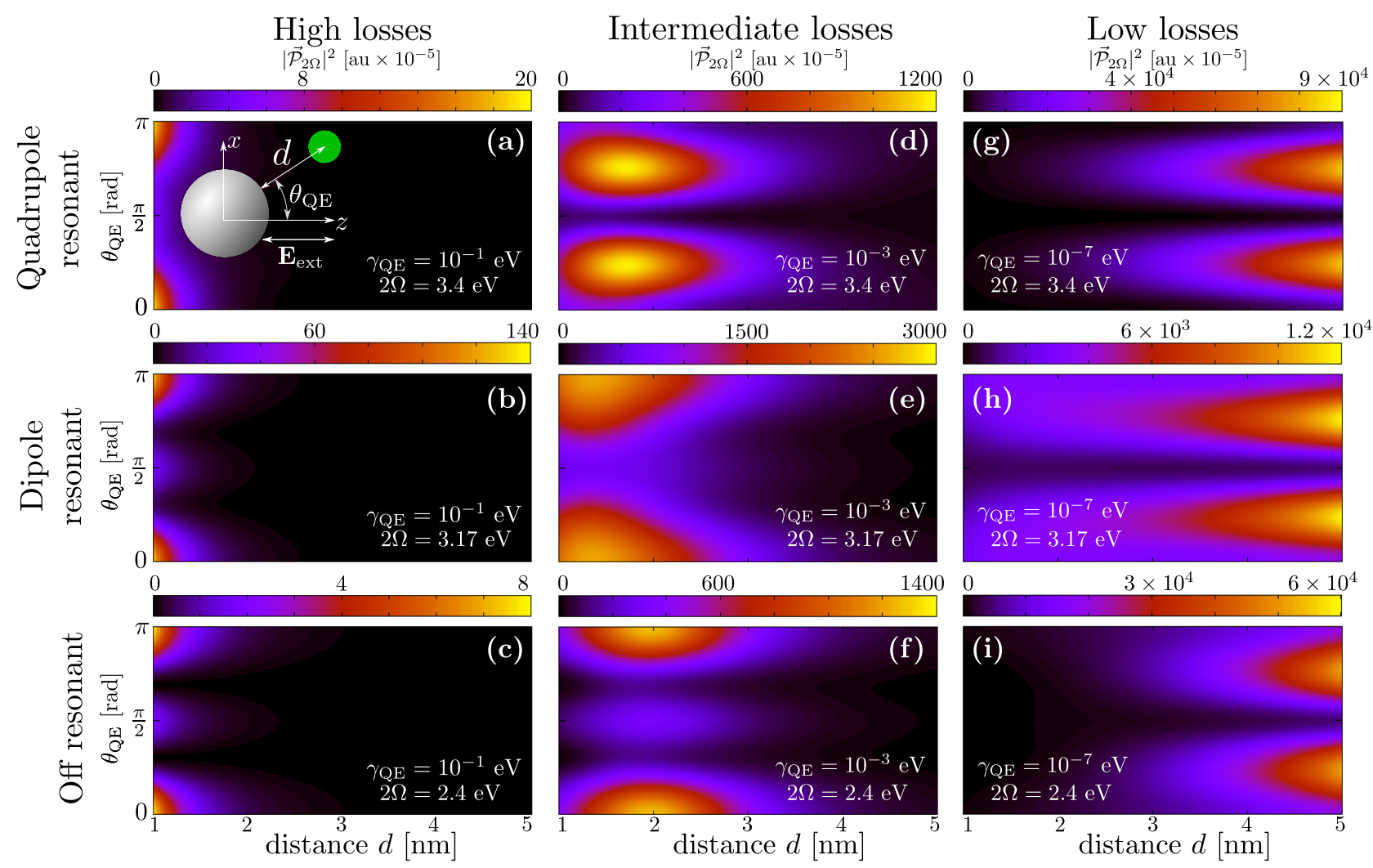

Figure 4: Second-harmonic response $\left|\overrightarrow{\mathcal{P}}_{2 \Omega}\right|^{2}$ of the coupled MNP-QE system illuminated with an incident $z$ polarized plane wave with frequency $\Omega$ and average intensity $I_{0}=10^{10} \mathrm{~W} \mathrm{~cm}^{-2}$ for different positions of the QE in the $(x, y)$-plane. The distance is measured from the surface of the MNP, and the QE is placed at a point given by $\left(d, \theta_{\mathrm{QE}}\right)$. The polar angle $\theta_{\mathrm{QE}}$ is measured with respect to the symmetry $z$-axis parallel to the electric field vector of the incident electromagnetic plane wave. For a sketch of the geometry, see Figure 1 and panel (a) of the present figure. The panels of the figure correspond to the results obtained for different values of the fundamental frequency $\Omega$ (rows) and intrinsic QE losses $\gamma_{\mathrm{OE}}$ (columns). We set $\Omega$ such that (i) the second harmonic is at resonance with the quadrupolar plasmon of the $\operatorname{MNP}(2 \Omega=3.4 \mathrm{eV}$, quadrupole resonant, top row); (ii) the second harmonic is at resonance with the dipolar plasmon of the $\mathrm{MNP}(2 \Omega=3.17 \mathrm{eV}$, dipole resonant, middle row); (iii) the fundamental frequency and the second harmonic are off-resonance with any mode of the MNP $(2 \Omega=2.4 \mathrm{eV}$, off resonant, bottom row). The choice of $\gamma_{\mathrm{QE}}$ illustrates the situations with high $\left(\gamma_{\mathrm{QE}}=10^{-1} \mathrm{eV}\right.$, left column), intermediate $\left(\gamma_{\mathrm{QE}}=10^{-3} \mathrm{eV}\right.$, center column), and low $\left(\gamma_{\mathrm{QE}}=10^{-7} \mathrm{eV}\right.$, right column) $\mathrm{QE}$ losses. For the values used at each panel, see also the labels in the figure.

$m$-polarized second-harmonic dipole $\overrightarrow{\mathcal{P}}_{2 \Omega}$ induced in the total system with the intensity $I_{0}$ of the incident plane wave) is $\left|\alpha_{\hat{\mathbf{m}}}^{(2)}(2 \Omega ; \Omega, \Omega)\right|=5.1 \times 10^{5}$ au and $\left|\alpha_{\hat{\mathbf{m}}}^{(2)}(2 \Omega ; \Omega, \Omega)\right|=2.3 \times 10^{6}$ au for high and intermediate losses, respectively (see details in Supporting Information).

The most prominent feature revealed by the results in Figure 4 is however the key role played by the intrinsic QE losses in the efficiency of the SHG and its dependence on the QE position. In general, lower intrinsic losses allow to reach significantly larger SHG. Remarkably, with $\gamma_{\mathrm{QE}}$ ranging from high $\left(10^{-1} \mathrm{eV}\right)$ to intermediate $\left(10^{-3} \mathrm{eV}\right)$ and low $\left(10^{-7} \mathrm{eV}\right)$ losses, the character of the distance $d$-dependence of the SHG (within the studied distances) changes completely. While $\left|\overrightarrow{\mathcal{P}}_{2 \Omega}\right|^{2}$ is monotonously decreasing for large $\mathrm{QE}$ losses in the range of distances considered, it is maximized at $d \sim 1.5-2 \mathrm{~nm}$ for intermediate losses, and at $d \sim 15-20 \mathrm{~nm}$ for low losses (this last result is not shown in the figure because it falls outside the validity limit of the nonretarded approximation used in our calculations). As a further striking result, for low intrinsic QE losses, the resonant condition with the dipolar plasmon $2 \Omega=\omega_{\text {DP }}$ leads not to the largest but to the smallest SHG. The maximum SHG is obtained in this case for the QE resonant with the quadrupolar plasmon mode of the MNP and for off-resonant conditions, reaching a maximum second-order hyperpolarizability $\left|\alpha_{\hat{\mathbf{m}}}^{(2)}(2 \Omega ; \Omega, \Omega)\right|=1.4 \times 10^{7}$ au. Our results show that this second-order nonlinear hyperpolarizability can be 3 orders of magnitude stronger than that of a typical highly nonlinear material such as $\mathrm{BaTiO}_{3}$ of similar geometry and size (see Supporting Information). However, we note that the scaling of the SHG with the size of the nanoparticles may be different for the two systems. 
In order to understand these results, it is useful to consider the respective weights of the contributions to the total second-harmonic dipole from the $\mathrm{QE}, \overrightarrow{\mathcal{P}}_{2 \Omega}^{\mathrm{QE}}$, and from the MNP, $\mathcal{P}_{2 \Omega}^{\text {np }}$, where $\overrightarrow{\mathcal{P}}_{2 \Omega}=\overrightarrow{\mathcal{P}}_{2 \Omega}^{\mathrm{QE}}+\overrightarrow{\mathcal{P}}_{2 \Omega}^{\mathrm{np}}$. For simplicity, we discuss the case of the QE located at the $z$-axis, but the same arguments are valid for other geometries. From eq 8 one obtains

$$
\begin{aligned}
& \frac{\left|\overrightarrow{\mathcal{P}}_{2 \Omega}^{\mathrm{QE}}\right|}{\left|\overrightarrow{\mathcal{P}}_{2 \Omega}\right|}=\frac{1}{\left|1+\alpha_{\mathrm{np}}(2 \Omega) \hat{\mathbf{z}} \overleftrightarrow{G}_{0}(\mathbf{R}) \hat{\mathbf{z}}\right|}, \\
& \frac{\left|\overrightarrow{\mathcal{P}}_{2 \Omega}^{\mathrm{np}}\right|}{\left|\overrightarrow{\mathcal{P}}_{2 \Omega}\right|}=\frac{\left|\alpha_{\mathrm{np}}(2 \Omega) \hat{\mathbf{z}} \overleftrightarrow{G}_{0}(\mathbf{R}) \hat{\mathbf{z}}\right|}{\left|1+\alpha_{\mathrm{np}}(2 \Omega) \hat{\mathbf{z}} \overleftrightarrow{G}_{0}(\mathbf{R}) \hat{\mathbf{z}}\right|} .
\end{aligned}
$$

These expressions are very useful since they do not depend on the characteristics of the QE. As shown in Figure $5 \mathrm{a}$ for the three excitation frequencies, at large distance $d$ the SHG is dominated by the dipole moment induced by the QE (dashed lines), while at short separation distances the dipole moment of the MNP provides the leading contribution (solid lines). Note that given the scaling of the MNP polarizability with the particle radius, $\alpha_{\mathrm{np}} \sim a^{3}$, and the dependence on the separation distance of the projection of the Green's dyadic in the near field, $\hat{\mathbf{z}} \overleftrightarrow{G}_{0} \hat{\mathbf{z}} \sim 1 /|a+d|^{3}$, the crossover region would move to larger distances $d$ with increasing MNP radius.

Along with the relative contributions of the nonlinear polarizations of the QE and of the MNP to the SHG, the response of the $Q E$ (probing the $2 \Omega$ near field) is an important characteristic of the system. The self-interaction due to the presence of the MNP modifies the total decay rate of the $\mathrm{QE}$, therefore once we have discussed the relative contribution of the $\mathrm{QE}$ and of the MNP to the SHG, it is useful to discuss how the decay rate of the QE changes due to this self-interaction with the MNP. The "effective" broadening (or, equivalently, total decay rate) of the QE resonance (Purcell effect) can be found from eq 8 and eq 10 .

$$
\gamma_{\mathrm{QE}}^{\prime}=\gamma_{\mathrm{QE}}+\alpha_{0} \operatorname{Im}\{\hat{\mathbf{z}} \stackrel{\leftrightarrow}{G}(\mathbf{R}, \mathbf{R}, 2 \Omega) \hat{\mathbf{z}}\} / 2 \Omega,
$$

where $\hat{\mathbf{z}} \overleftrightarrow{G} \hat{\mathbf{z}} \sim 1 /|\mathbf{R}|^{6}=1 /(a+d)^{6}$ in first approximation $(l=1$ in eq 12$)$. We show the self-interaction contribution to $\gamma_{\mathrm{QE}}^{\prime}$ (last term in eq 15 in Figure $5 \mathrm{p}$. Because of the frequency dependence of the MNP polarizability, this is largest when the second-harmonic frequency is resonant with the dipolar plasmon of the MNP and smallest for the off-resonance conditions. Importantly, the broadening $\gamma_{\mathrm{OE}}^{\prime}$ of the QE transition determines the maximum nonlinear polarization of the $\mathrm{QE}$ that can be reached at resonance with the second-harmonic near field of the MNP, $\omega_{0}=2 \Omega$.

We are now in a position to explain the main trends observed in Figure 4 Let us consider first the case of large intrinsic losses $\gamma_{\mathrm{QE}}=10^{-1} \mathrm{eV}$ dominating the decay of the QE over the extra losses introduced by the (a)

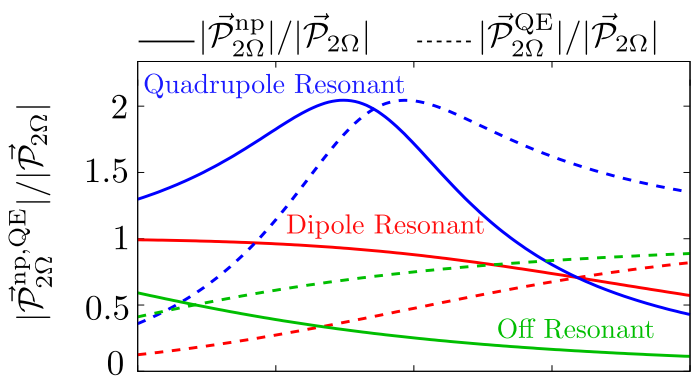

(b)

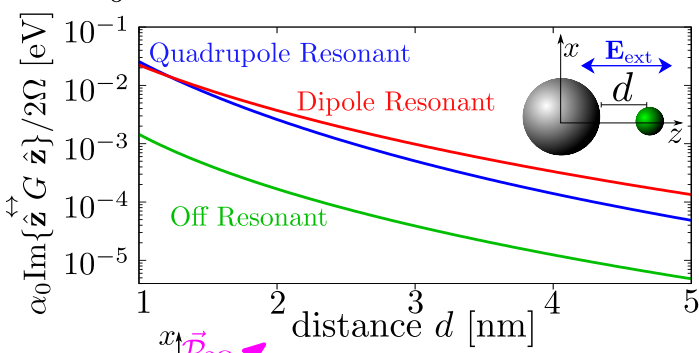

(c)

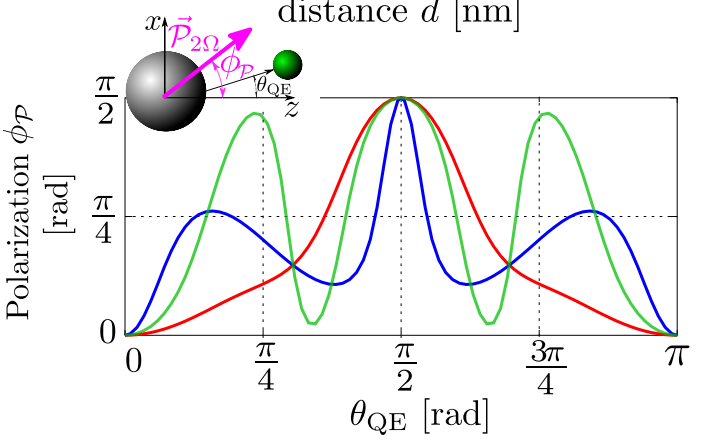

Figure 5: (a) Relative contributions of the MNP: $\left|\overrightarrow{\mathcal{P}}_{2 \Omega}^{\text {np }}\right| /\left|\overrightarrow{\mathcal{P}}_{2 \Omega}\right|$ (solid lines), and of the QE: $\left|\overrightarrow{\mathcal{P}}_{2 \Omega}^{\mathrm{QE}}\right| /\left|\overrightarrow{\mathcal{P}}_{2 \Omega}\right|$ (dashed lines) to the total nonlinear dipole of the system at the second-harmonic frequency. Results are shown as a function of the MNP-QE distance $d$. The QE is located at the $z$-axis $\left(\theta_{\mathrm{QE}}=0\right)$. (b) Additional broadening of the QE transition because of the interaction with the MNP. The quantity $\alpha_{0} \operatorname{Im}\{\hat{\mathbf{z}} \overleftrightarrow{G}(\mathbf{R}, \mathbf{R}, 2 \Omega) \hat{\mathbf{z}}\} / 2 \Omega$ is shown as a function of the MNP-QE distance $d$. (c) Dependence of the polarization direction of the total emitting dipole $\phi_{\mathcal{P}}$ on the angular position $\theta_{\mathrm{QE}}$ of the QE. The QE distance from the MNP surface is fixed to $d=2 \mathrm{~nm}$, and $\gamma_{\mathrm{QE}}=10^{-3} \mathrm{eV} \cdot \theta_{\mathrm{QE}}=0$ corresponds to the $\mathrm{QE}$ located at the $z$-axis, and $\theta_{\mathrm{QE}}=\pi / 2$ corresponds to the $\mathrm{QE}$ located at the $x$-axis (see inset). In all the panels of the figure we consider three different excitation frequencies corresponding to $\Omega=1.7 \mathrm{eV}$ (second harmonic at resonance with the quadrupole plasmon of the MNP, blue lines), $\Omega=1.585 \mathrm{eV}$ (second harmonic at resonance with the dipolar plasmon of the MNP, red lines), and $\Omega=1.2 \mathrm{eV}$ (off-resonant conditions, green lines).

self-interaction, so that the "effective" broadening remains unchanged, $\gamma_{\mathrm{OE}}^{\prime} \simeq \gamma_{\mathrm{QE}}$ (see Figure $5 p$ ). The $\mathrm{QE}$ dipole at the second-harmonic frequency can then be estimated from $\overrightarrow{\mathcal{P}}_{2 \Omega}^{\mathrm{QE}} \propto \mathbf{E}_{\text {ind }}^{\mathrm{TDDT}}(\mathbf{R}, 2 \Omega) / \gamma_{\mathrm{QE}}$. The near field at the second-harmonic frequency induced by the MNP in response to the incident field is of quadrupolar character so that $\mathbf{E}_{\text {ind }}^{\mathrm{TDDF}} \sim 1 /|\mathbf{R}|^{4}$, and correspond- 
ingly $\overrightarrow{\mathcal{P}}_{2 \Omega}^{\mathrm{QE}} \propto 1 /|\mathbf{R}|^{4}$. Further, the second-harmonic dipole of the MNP resulting from the interaction with the QE can be obtained from eq 8 . This is given by $\overrightarrow{\mathcal{P}}_{2 \Omega}^{\text {np }}=\alpha_{\text {np }}(2 \Omega) \overleftrightarrow{G}_{0} \overrightarrow{\mathcal{P}}_{2 \Omega}^{\mathrm{QE}}$, which results in the following dependence of the second-harmonic dipole with the QE position: $\overrightarrow{\mathcal{P}}_{2 \Omega}^{\text {np }} \propto 1 /|\mathbf{R}|^{7}$. Therefore, the maximum total nonlinear dipole $\overrightarrow{\mathcal{P}}_{2 \Omega}$ is reached for small $d$ (the MNP dominating regime), and it is monotonically decreasing with increasing $d$ as a consequence of the drop-off of both the second-harmonic near field and the MNP-QE interaction. Since the emission is maximum for short distances where it is dominated by the nonlinear dipole moment of the MNP, the power emitted to the far field is enhanced when the second-harmonic matches the dipolar plasmon, i.e., when the MNP polarizability is largest.

Let us consider now the case of low intrinsic losses $\gamma_{\mathrm{QE}}=10^{-7} \mathrm{eV}$ such that the decay of the QE polarization is determined by the self-interaction for all the distances in the figures: $\gamma_{\mathrm{OE}}^{\prime} \simeq \alpha_{0} \operatorname{Im}\{\hat{\mathbf{z}} \stackrel{\leftrightarrow}{G}(\mathbf{R}, \mathbf{R}, 2 \Omega) \hat{\mathbf{z}}\} / 2 \Omega$ (plotted in Figure $5 \mathrm{~b}$ ). The nonlinear dipole of the $\mathrm{QE}$ at the second-harmonic frequency can then be estimated from $\overrightarrow{\mathcal{P}}_{2 \Omega}^{\mathrm{QE}} \propto \mathbf{E}_{\text {ind }}^{\mathrm{TDDF}}(\mathbf{R}, 2 \Omega) / \gamma_{\mathrm{QE}}^{\prime}$. The dependence of $\overrightarrow{\mathcal{P}}_{2 \Omega}^{\mathrm{QE}}$ on the separation distance $d$ is thus governed by that of the self interaction $\gamma_{\mathrm{QE}}^{\prime} \sim 1 /|\mathbf{R}|^{6}$ and that of the quadrupole near field at the second harmonic $\mathbf{E}_{\text {ind }}^{\mathrm{TDDF}} \sim 1 /|\mathbf{R}|^{4}$. As a result, $\overrightarrow{\mathcal{P}}_{2 \Omega}^{\mathrm{QE}} \propto|\mathbf{R}|^{2}$, while $\overrightarrow{\mathcal{P}}_{2 \Omega}^{\text {np }} \propto 1 /|\mathbf{R}|$. The largest nonlinear dipole can finally be reached at large $|\mathbf{R}|$ owing to the polarization of the QE. The system is in the regime where the emission from the QE dominates and the SHG intensity increases as $\sim|\mathbf{R}|^{4}$. Additionally, since $\operatorname{Im}\{\hat{\mathbf{z}} \overleftrightarrow{G}(\mathbf{R}, \mathbf{R}, \omega) \hat{\mathbf{z}}\}$ is maximum at resonance with the dipolar plasmon of the MNP, setting $2 \Omega=\omega_{\text {DP }}$ leads to a larger width of the QE resonance (larger losses) and thus to smaller SHG. Thus, the largest nonlinear signal is obtained in this regime for the second harmonic in resonance with the quadrupolar mode of the MNP and for off-resonance conditions. Apart from the losses introduced by the MNP, the second-harmonic near field $\mathbf{E}_{\text {ind }}^{\mathrm{TDDT}}(\mathbf{R}, \omega=2 \Omega)$ induced by the individual MNP is stronger for the second harmonic matching the quadrupolar plasmon resonance of the $\mathrm{MNP},{ }^{92} 2 \Omega=\omega_{\mathrm{QP}}=3.4 \mathrm{eV}$, which finally results in the largest SHG as shown in Figure $4 \mathrm{~g}$. Interestingly, the reduction of the second-harmonic signal at small $|\mathbf{R}|$ because of the self-interaction terms observed here has a similar physical origins as the well-known effect of the QE luminescence quenching in the linear case. .8487

For intermediate losses $\gamma_{\mathrm{QE}}=10^{-3} \mathrm{eV}$, the "effective" broadening of the $\mathrm{QE}$ is given by the self-interaction at small distances $d$ from the surface and saturates to the intrinsic value $\gamma_{\mathrm{QE}}$ at larger distances (see Figure $5 \mathrm{p}$ ). As a consequence, by increasing $d$ the induced QE polarization at the second harmonic changes its distance dependence from $\overrightarrow{\mathcal{P}}_{2 \Omega}^{\mathrm{QE}} \propto|\mathbf{R}|^{2}$ to $\overrightarrow{\mathcal{P}}_{2 \Omega}^{\mathrm{QE}} \propto 1 /|\mathbf{R}|^{4}$ lead- ing to a maximum of the SGH for the $d=1.5-2 \mathrm{~nm}$ distance range.

To close this section it is worthwhile to point out an attractive possibility to control the polarization of the light emitted at the second-harmonic frequency, owing to the quadrupolar character of the second-harmonic near field of the MNP induced by the plane-wave excitation. In Figure 55 we analyze the direction of polarization of the total second-harmonic dipole of the system for different positions of the QE. The direction of polarization of the nonlinear dipole is defined by the angle $\phi_{\mathcal{P}}$, measured between $\overrightarrow{\mathcal{P}}_{2 \Omega}$ and the positive direction of the $z$-axis. The position of the $Q E$ is given by the corresponding angle $\theta_{\mathrm{QE}}$ (see inset). The $\mathrm{QE}$ is placed at different $\theta_{\mathrm{QE}}$ while keeping a fixed distance $d=2 \mathrm{~nm}$ from the MNP surface. The calculations are performed considering intermediate intrinsic losses $\gamma_{\mathrm{QE}}=10^{-3} \mathrm{eV}$, but the same qualitative behavior is found for other cases.

When the $\mathrm{QE}$ is located at $\theta_{\mathrm{QE}}=0$ or $\theta_{\mathrm{QE}}=\pi$, corresponding to the polarization direction of the external laser field, the total emitting dipole is z-polarized $\left(\phi_{\mathcal{P}}=0\right)$, i.e., it is parallel to the excitation laser. In contrast, when the $\mathrm{QE}$ is placed at $\theta_{\mathrm{QE}}=\pi / 2$ the induced dipole is $x$-polarized $\left(\phi_{\mathcal{P}}=\pi / 2\right)$. This implies a full polarization conversion of the second-harmonic radiation with respect to the fundamental wave. This result is a robust consequence of the symmetry of the system, as discussed in previous sections. When the QE is located at the $z$-axis, the system has a rotation symmetry with respect to the $z$-axis, thus no $x$ - or $y$-polarized SHG can be produced. In a similar way, when the QE is located at the $x$-axis, the system is symmetric with respect to the $(x, y)$-plane, and therefore the $z$-polarized SHG is forbidden.

\section{Summary and Conclusions}

We have studied how the coupling of a QE with a centrosymmetric MNP alters the nonlinear response of this compound system and enables otherwise forbidden SHG. Using TDDFT simulations we have shown that when the second harmonic of the fundamental frequency is resonant with the transition frequency of the quantum emitter, the latter plays the role of an optical resonator that scatters the local second-harmonic near field created by the MNP into the far field.

For the present model, the TDDFT calculations reveal the following three-step scenario of the SHG process: first, the MNP generates a second-harmonic near field in response to the incident radiation, second, the QE is resonantly excited at $2 \Omega$ by this near field, and finally, the QE emits in the presence of the MNP. We would like to stress however that the generalization of this mechanism to other systems has to be done carefully considering the relevant characteristics of the QE and the plasmonic nanoobject. In particular, the polarization of the $\mathrm{QE}$ at the fundamental frequency can also lead to 
an appreciable nonlinear response of the MNP as would be, for instance, the case of a small MNP and a strongly polarizable QE.

The insights provided by the TDDFT calculations allowed to develop a semiclassical model of the secondharmonic response of the MNP-QE system. Using the semiclassical model, we have demonstrated that the efficiency of the SHG, its dependence on the position of the $\mathrm{QE}$, and its dependence on the frequency match between the plasmon modes of the MNP and the QE transition is determined by the relative importance of the intrinsic losses of the QE and the broadening of the QE transition due to the self-interaction via the MNP. We have shown that it is possible to obtain orders of magnitude stronger SHG by reducing the intrinsic losses of the QE. Owing to the large SHG obtained in the present system, we believe that the effect of the QE will persist in a practical experimental situation where the MNP geometry imperfections and the substrate may produce a 'background' SHG.

Finally, we have demonstrated the possibility to control the polarization of the light emitted at the second harmonic. We have shown that the polarization of the total second-harmonic dipole of the MNP-QE system depends on the position of the QE with respect to the axis defined by the polarization of the incident light. In particular, this allows to obtain a full polarization conversion where the dipole emitting at the second harmonic is perpendicular to the illuminating field.

Although the results shown in this work are obtained for a model spherical nanoparticle and a structureless $\mathrm{QE}$, the qualitative conclusions driven here stem from the robust phenomenon of generation of even-harmonic signal in the near field close to the MNP surface, from general symmetry constraints, and from the physics of optical resonators interacting with plasmonic nanoparticles. It thus appears from our findings that the hybrid MNP-QE structure can be a promising platform for enhancing second-harmonic generation and probing the nonlinear near fields of metallic nanostructures.

Acknowledgement A.B., R.E., and J.A. acknowledge the National Project FIS2016-80174-P from the MICINN and Project PI2017-30 and Grant No. IT116419 for research groups of the Basque University system from the Department of Education of the Basque Government. A.B also thanks Dr. Garikoitz Aguirregabiria for valuable technical advice on the TDDFT simulations of metallic nanoparticles and the Department of Education of the Basque Government for a predoctoral fellowship (Grant No. PRE2017_1_0267). A.G.B. gratefully acknowledges the hospitality of the Donostia International Physics Center.

\section{Supporting Information Avail- able}

TDDFT calculations to identify the dipolar and quadrupolar plasmon resonances of the individual MNP, details on how we determine the main physical mechanism behind the SHG, the explanation of the filtering procedure applied to the semiclassical results of part $b$ and $c$ of Figure 3 for comparison with TDDFT, relationship between the results obtained under finite Gaussian pulse and plane-wave excitation, and the calculation of the second-order nonlinear hyperpolarizability. This material is available free of charge via the Internet at http:// pubs.acs.org

\section{References}

(1) Kauranen, M.; Zayats, A. V. Nonlinear plasmonics. Nature photonics 2012, 6, 737 .

(2) Krasavin, A. V.; Ginzburg, P.; Zayats, A. V. In Quantum Photonics: Pioneering Advances and Emerging Applications; Boyd, R. W., Lukishova, S. G., Zadkov, V. N., Eds.; Springer International Publishing: Cham, 2019; pp 267-316.

(3) Bouhelier, A.; Beversluis, M.; Hartschuh, A.; Novotny, L. NearField Second-Harmonic Generation Induced by Local Field Enhancement. Phys. Rev. Lett. 2003, 90, 013903.

(4) Anceau, C.; Brasselet, S.; Zyss, J.; Gadenne, P. Local secondharmonic generation enhancement on gold nanostructures probed by two-photon microscopy. Optics letters 2003, 28, 713715.

(5) Li, K.; Stockman, M. I.; Bergman, D. J. Enhanced second harmonic generation in a self-similar chain of metal nanospheres. Phys. Rev. B 2005, 72, 153401.

(6) Palomba, S.; Danckwerts, M.; Novotny, L. Nonlinear plasmonics with gold nanoparticle antennas. Journal of Optics A: Pure and Applied Optics 2009, 11, 114030.

(7) Ciracì, C.; Poutrina, E.; Scalora, M.; Smith, D. R. Secondharmonic generation in metallic nanoparticles: clarification of the role of the surface. Physical Review B 2012, 86, 115451.

(8) Butet, J.; Brevet, P.-F.; Martin, O. J. Optical second harmonic generation in plasmonic nanostructures: from fundamental principles to advanced applications. ACS nano 2015, 9, 1054510562.

(9) Celebrano, M.; Wu, X.; Baselli, M.; Großmann, S.; Biagioni, P.; Locatelli, A.; De Angelis, C.; Cerullo, G.; Osellame, R.; Hecht, B.; Duò, L.; Ciccacci, F.; Finazzi, M. Mode matching in multiresonant plasmonic nanoantennas for enhanced second harmonic generation. Nature nanotechnology 2015, 10, 412.

(10) Butet, J.; Bernasconi, G. D.; Petit, M.; Bouhelier, A.; Yan, C.; Martin, O. J. F.; Cluzel, B.; Demichel, O. Revealing a Mode Interplay That Controls Second-Harmonic Radiation in Gold Nanoantennas. ACS Photonics 2017, 4, 2923-2929.

(11) Bernasconi, G. D.; Butet, J.; Martin, O. J. Dynamics of SecondHarmonic Generation in a Plasmonic Silver Nanorod. ACS Photonics 2018, 5, 3246-3254.

(12) Husu, H.; Siikanen, R.; Makitalo, J.; Lehtolahti, J.; Laukkanen, J.; Kuittinen, M.; Kauranen, M. Metamaterials with tailored nonlinear optical response. Nano letters 2012, 12, 673-677.

(13) Aguirregabiria, G.; Marinica, D. C.; Esteban, R.; Kazansky, A. K.; Aizpurua, J.; Borisov, A. G. Electric Field-Induced High Order Nonlinearity in Plasmonic Nanoparticles Retrieved with Time-Dependent Density Functional Theory. ACS Photonics 2017, 4, 613-620.

(14) Aguirregabiria, G.; Marinica, D. C.; Esteban, R.; Kazansky, A. K.; Aizpurua, J.; Borisov, A. G. Role of electron tunnel- 
ing in the nonlinear response of plasmonic nanogaps. Physical Review B 2018, 97, 115430.

(15) Berthelot, J.; Bachelier, G.; Song, M.; Rai, P.; Des Francs, G. C.; Dereux, A.; Bouhelier, A. Silencing and enhancement of secondharmonic generation in optical gap antennas. Optics express 2012, 20, 10498-10508.

(16) Cai, W.; Vasudev, A. P.; Brongersma, M. L. Electrically controlled nonlinear generation of light with plasmonics. Science 2011, 333, 1720-1723.

(17) Kang, L.; Cui, Y.; Lan, S.; Rodrigues, S. P.; Brongersma, M. L.; Cai, W. Electrifying photonic metamaterials for tunable nonlinear optics. Nature communications 2014, 5, 4680.

(18) Czaplicki, R.; Husu, H.; Siikanen, R.; Mäkitalo, J.; Kauranen, M.; Laukkanen, J.; Lehtolahti, J.; Kuittinen, M. Enhancement of second-harmonic generation from metal nanoparticles by passive elements. Physical review letters 2013, 110, 093902.

(19) Ginzburg, P.; Krasavin, A.; Sonnefraud, Y.; Murphy, A.; Pollard, R. J.; Maier, S. A.; Zayats, A. V. Nonlinearly coupled localized plasmon resonances: Resonant second-harmonic generation. Phys. Rev. B 2012, 86, 085422.

(20) Slablab, A.; Xuan, L. L.; Zielinski, M.; de Wilde, Y.; Jacques, V.; Chauvat, D.; Roch, J.-F. Second-harmonic generation from coupled plasmon modes in a single dimer of gold nanospheres. Opt. Express 2012, 20, 220-227.

(21) Thyagarajan, K.; Butet, J.; Martin, O. J. F. Augmenting Second Harmonic Generation Using Fano Resonances in Plasmonic Systems. Nano Letters 2013, 13, 1847-1851.

(22) Ginzburg, P.; Krasavin, A. V.; Wurtz, G. A.; Zayats, A. V. Nonperturbative Hydrodynamic Model for Multiple Harmonics Generation in Metallic Nanostructures. ACS Photonics 2015, 2, 8-13.

(23) Black, L.-J.; Wiecha, P. R.; Wang, Y.; de Groot, C. H.; Paillard, V.; Girard, C.; Muskens, O. L.; Arbouet, A. Tailoring Second-Harmonic Generation in Single L-Shaped Plasmonic Nanoantennas from the Capacitive to Conductive Coupling Regime. ACS Photonics 2015, 2, 1592-1601.

(24) Gennaro, S. D.; Rahmani, M.; Giannini, V.; Aouani, H.; Sidiropoulos, T. P. H.; Navarro-Cia, M.; Maier, S. A.; Oulton, R. F. The Interplay of Symmetry and Scattering Phase in Second Harmonic Generation from Gold Nanoantennas. Nano Letters 2016, 16, 5278-5285.

(25) Krasavin, A. V.; Ginzburg, P.; Zayats, A. V. Free-electron Optical Nonlinearities in Plasmonic Nanostructures: A Review of the Hydrodynamic Description. Laser \& Photonics Reviews 2018, 12, 1700082.

(26) Thyagarajan, K.; Rivier, S.; Lovera, A.; Martin, O. J. Enhanced second-harmonic generation from double resonant plasmonic antennae. Optics express 2012, 20, 12860-12865.

(27) Aouani, H.; Navarro-Cia, M.; Rahmani, M.; Sidiropoulos, T. P. H.; Hong, M.; Oulton, R. F.; Maier, S. A. Multiresonant Broadband Optical Antennas As Efficient Tunable Nanosources of Second Harmonic Light. Nano Letters 2012, 12, 4997-5002.

(28) Hasan, S. B.; Lederer, F.; Rockstuhl, C. Nonlinear plasmonic antennas. Materials Today 2014, 17, $478-485$

(29) Metzger, B.; Gui, L.; Fuchs, J.; Floess, D.; Hentschel, M.; Giessen, H. Strong Enhancement of Second Harmonic Emission by Plasmonic Resonances at the Second Harmonic Wavelength. Nano Letters 2015, 15, 3917-3922.

(30) Linnenbank, H.; Grynko, Y.; Förstner, J.; Linden, S. Second harmonic generation spectroscopy on hybrid plasmonic/dielectric nanoantennas. Light: Science and Applications 2016, 5, e16013.

(31) Yang, K.-Y.; Butet, J.; Yan, C.; Bernasconi, G. D.; Martin, O. J. Enhancement mechanisms of the second harmonic generation from double resonant aluminum nanostructures. ACS Photonics 2017, 4, 1522-1530.

(32) Metzger, B.; Hentschel, M.; Giessen, H. Probing the near-field of second-harmonic light around plasmonic nanoantennas. Nano letters 2017, 17, 1931-1937.

(33) Zhang, R.; Zhang, Y.; Dong, Z. C.; Jiang, S.; Zhang, C.; Chen, L. G.; Zhang, L.; Liao, Y.; Aizpurua, J.; Luo, Y.; Luo, Y.;
Yang, J. L.; Hou, J. G. Chemical mapping of a single molecule by plasmon-enhanced Raman scattering. Nature 2013, 498, 82.

(34) Song, P.; Nordlander, P.; Gao, S. Quantum mechanical study of the coupling of plasmon excitations to atomic-scale electron transport. The Journal of chemical physics 2011, 134, 074701.

(35) Tan, S. F.; Wu, L.; Yang, J. K.; Bai, P.; Bosman, M.; Nijhuis, C. A Quantum plasmon resonances controlled by molecular tunnel junctions. Science 2014, 343, 1496-1499.

(36) Esteban, R.; Aizpurua, J.; Bryant, G. W. Strong coupling of single emitters interacting with phononic infrared antennae. New Journal of Physics 2014, 16, 013052.

(37) Kulkarni, V.; Manjavacas, A. Quantum effects in charge transfer plasmons. ACS Photonics 2015, 2, 987-992.

(38) Neuman, T.; Esteban, R.; Casanova, D.; García-Vidal, F.; Aizpurua, J. Coupling of molecular emitters and plasmonic cavities beyond the point-dipole approximation. Nano letters 2018, 18, 2358-2364

(39) Marinica, D.; Lourenço-Martins, H.; Aizpurua, J.; Borisov, A. G. Plexciton quenching by resonant electron transfer from quantum emitter to metallic nanoantenna. Nano letters 2013, 13, 59725978.

(40) Galego, J.; Garcia-Vidal, F.; Feist, J. Cavity-induced modifications of molecular structure in the strong-coupling regime. Physical Review X 2015, 5, 041022.

(41) Benz, F.; Tserkezis, C.; Herrmann, L. O.; De Nijs, B.; Sanders, A.; Sigle, D. O.; Pukenas, L.; Evans, S. D.; Aizpurua, J.; Baumberg, J. J. Nanooptics of molecular-shunted plasmonic nanojunctions. Nano letters 2014, 15, 669-674.

(42) Singh, M. R. Enhancement of the second-harmonic generation in a quantum dot-metallic nanoparticle hybrid system. Nanotechnology 2013, 24, 125701.

(43) Turkpence, D.; Akguc, G. B.; Bek, A.; Tasgin, M. E. Engineering nonlinear response of nanomaterials using Fano resonances. Journal of Optics 2014, 16, 105009.

(44) Cox, J. D.; de Abajo, F. J. G. Nonlinear Atom-Plasmon Interactions Enabled by Nanostructured Graphene. Physical review letters 2018, 121, 257403.

(45) Gross, E.; Kohn, W. In Density Functional Theory of ManyFermion Systems; Löwdin, P.-O., Ed.; Advances in Quantum Chemistry; Academic Press, 1990; Vol. 21; pp 255 - 291.

(46) Marques, M.; Gross, E. Time-Dependent Density Functional Theory. Annual Review of Physical Chemistry 2004, 55, 427-455.

(47) Shaviv, E.; Banin, U. Synergistic Effects on Second Harmonic Generation of Hybrid CdSe- Au Nanoparticles. ACS nano 2010, 4, 1529-1538.

(48) Linnenbank, H.; Linden, S. Second harmonic generation spectroscopy on second harmonic resonant plasmonic metamaterials. Optica 2015, 2, 698-701.

(49) Brack, M. The physics of simple metal clusters: self-consistent jellium model and semiclassical approaches. Reviews of modern physics 1993, 65, 677 .

(50) Ekardt, W. Dynamical polarizability of small metal particles: self-consistent spherical jellium background model. Physical review letters 1984, 52, 1925.

(51) Koskinen, M.; Lipas, P.; Manninen, M. Electron-gas clusters: the ultimate jellium model. Zeitschrift für Physik D Atoms, Molecules and Clusters 1995, 35, 285-297.

(52) Pitarke, J.; Silkin, V.; Chulkov, E.; Echenique, P. Theory of surface plasmons and surface-plasmon polaritons. Reports on progress in physics 2006, 70, 1.

(53) Krüger, M.; Lemell, C.; Wachter, G.; Burgdörfer, J.; Hommelhoff, P. Attosecond physics phenomena at nanometric tips. Journal of Physics B: Atomic, Molecular and Optical Physics

(54) Pastore, M.; Mosconi, E.; De Angelis, F.; GrÃd'tzel, M. A Computational Investigation of Organic Dyes for Dye-Sensitized Solar Cells: Benchmark, Strategies, and Open Issues. The Journal of Physical Chemistry C 2010, 114, 7205-7212.

(55) Zuloaga, J.; Prodan, E.; Nordlander, P. Quantum Description of the Plasmon Resonances of a Nanoparticle Dimer. Nano Letters 
2009, 9, 887-891.

(56) Marinica, D.; Kazansky, A.; Nordlander, P.; Aizpurua, J.; Borisov, A. G. Quantum Plasmonics: Nonlinear Effects in the Field Enhancement of a Plasmonic Nanoparticle Dimer. Nano Letters 2012, 12, 1333-1339.

(57) Scholl, J. A.; Garcia-Etxarri, A.; Koh, A. L.; Dionne, J. A. Observation of Quantum Tunneling between Two Plasmonic Nanoparticles. Nano Letters 2013, 13, 564-569.

(58) Zhang, P.; Feist, J.; Rubio, A.; García-González, P.; GarcíaVidal, F. Ab initio nanoplasmonics: The impact of atomic structure. Physical Review B 2014, 90, 161407.

(59) Varas, A.; García-González, P.; Feist, J.; García-Vidal, F.; Rubio, A. Quantum plasmonics: from jellium models to ab initio calculations. Nanophotonics 2016, 5, 409-426.

(60) Barbry, M.; Koval, P.; Marchesin, F.; Esteban, R.; Borisov, A. G.; Aizpurua, J.; Sánchez-Portal, D. Atomistic near-field nanoplasmonics: reaching atomic-scale resolution in nanooptics. Nano letters 2015, 15, 3410-3419.

(61) Jones, R. O. Density functional theory: Its origins, rise to prominence, and future. Rev. Mod. Phys. 2015, 87, 897-923.

(62) Leforestier, C.; Bisseling, R. H.; Cerjan, C.; Feit, M. D.; Friesner, R.; Guldberg, A.; Hammerich, A.; Jolicard, G.; Karrlein, W.; Meyer, H.-D.; Lipkin, N.; Roncero, O.; Kosloff, R. A comparison of different propagation schemes for the time dependent Schrödinger equation. Journal of Computational Physics 1991, 94, 59-80.

(63) Gunnarsson, O.; Lundqvist, B. I. Exchange and correlation in atoms, molecules, and solids by the spin-density-functional formalism. Physical Review B 1976, 13, 4274.

(64) Wijewardane, H. O.; Ullrich, C. A. Time-Dependent KohnSham Theory with Memory. Phys. Rev. Lett. 2005, 95, 086401.

(65) Vignale, G.; Kohn, W. Current-Dependent ExchangeCorrelation Potential for Dynamical Linear Response Theory. Phys. Rev. Lett. 1996, 77, 2037-2040.

(66) Ullrich, C. A. Time-dependent density-functional theory: concepts and applications; Oxford University Press, 2013.

(67) Letfullin, R. R.; Joenathan, C.; George, T. F.; Zharov, V. P. Laserinduced explosion of gold nanoparticles: potential role for nanophotothermolysis of cancer. Nanomedicine 2006, 1, 473-480, PMID: 17716149.

(68) Fennel, T.; Meiwes-Broer, K.-H.; Tiggesbäumker, J.; Reinhard, P.-G.; Dinh, P. M.; Suraud, E. Laser-driven nonlinear cluster dynamics. Rev. Mod. Phys. 2010, 82, 1793-1842.

(69) Danckwerts, M.; Novotny, L. Optical Frequency Mixing at Coupled Gold Nanoparticles. Phys. Rev. Lett. 2007, 98, 026104.

(70) Boyd, R. W. Nonlinear optics; Elsevier, 2003.

(71) Shen, Y. Surface properties probed by second-harmonic and sum-frequency generation. Nature 1989, 337, 519.

(72) Rudnick, J.; Stern, E. A. Second-Harmonic Radiation from Metal Surfaces. Phys. Rev. B 1971, 4, 4274-4290.

(73) Sipe, J. E.; So, V. C. Y.; Fukui, M.; Stegeman, G. I. Analysis of second-harmonic generation at metal surfaces. Phys. Rev. B 1980, 21, 4389-4402.

(74) Zavelani-Rossi, M.; Celebrano, M.; Biagioni, P.; Polli, D.; Finazzi, M.; Duo, L.; Cerullo, G.; Labardi, M.; Allegrini, M.; Grand, J.; Adam, P.-M. Near-field second-harmonic generation in single gold nanoparticles. Applied physics letters 2008, 92, 093119.

(75) Butet, J.; Bachelier, G.; Russier-Antoine, I.; Jonin, C.; Benichou, E.; Brevet, P.-F. Interference between selected dipoles and octupoles in the optical second-harmonic generation from spherical gold nanoparticles. Physical review letters 2010, 105, 077401.

(76) Butet, J.; Thyagarajan, K.; Martin, O. J. Ultrasensitive optical shape characterization of gold nanoantennas using second harmonic generation. Nano letters 2013, 13, 1787-1792.

(77) Hua, X. M.; Gersten, J. I. Theory of second-harmonic generation by small metal spheres. Phys. Rev. B 1986, 33, 3756-3764.

(78) Dadap, J. I.; Shan, J.; Eisenthal, K. B.; Heinz, T. F. Second-
Harmonic Rayleigh Scattering from a Sphere of Centrosymmetric Material. Phys. Rev. Lett. 1999, 83, 4045-4048.

(79) Dadap, J. I.; Shan, J.; Heinz, T. F. Theory of optical secondharmonic generation from a sphere of centrosymmetric material: small-particle limit. J. Opt. Soc. Am. B 2004, 21, 1328-1347.

(80) Russier-Antoine, I.; Benichou, E.; Bachelier, G.; Jonin, C.; Brevet, P. F. Multipolar Contributions of the Second Harmonic Generation from Silver and Gold Nanoparticles. The Journal of Physical Chemistry C 2007, 111, 9044-9048.

(81) Bachelier, G.; Russier-Antoine, I.; Benichou, E.; Jonin, C.; Brevet, P.-F. Multipolar second-harmonic generation in noble metal nanoparticles. J. Opt. Soc. Am. B 2008, 25, 955-960.

(82) Bachelier, G.; Butet, J.; Russier-Antoine, I.; Jonin, C.; Benichou, E.; Brevet, P.-F. Origin of optical second-harmonic generation in spherical gold nanoparticles: Local surface and nonlocal bulk contributions. Phys. Rev. B 2010, 82, 235403.

(83) Morawitz, H. Self-coupling of a two-level system by a mirror. Physical Review 1969, 187, 1792.

(84) Carminati, R.; Greffet, J.-J.; Henkel, C.; Vigoureux, J.-M. Radiative and non-radiative decay of a single molecule close to a metallic nanoparticle. Optics Communications 2006, 261, 368375.

(85) Novotny, L.; Hecht, B. Principles of nano-optics; Cambridge university press, 2012.

(86) Jackson, J. D. Classical electrodynamics. 1999.

(87) Delga, A.; Feist, J.; Bravo-Abad, J.; Garcia-Vidal, F. Quantum emitters near a metal nanoparticle: strong coupling and quenching. Physical review letters 2014, 112, 253601.

(88) Ford, G. W.; Weber, W. H. Electromagnetic interactions of molecules with metal surfaces. Physics Reports 1984, 113, 195287.

(89) Yannouleas, C.; Broglia, R. Landau damping and wall dissipation in large metal clusters. Annals of Physics 1992, 217, 105 141.

(90) Teperik, T. V.; Nordlander, P.; Aizpurua, J.; Borisov, A. G. Robust subnanometric plasmon ruler by rescaling of the nonlocal optical response. Physical review letters 2013, 110, 263901.

(91) Toscano, G.; Straubel, J.; Kwiatkowski, A.; Rockstuhl, C.; Evers, F.; Xu, H.; Mortensen, N. A.; Wubs, M. Resonance shifts and spill-out effects in self-consistent hydrodynamic nanoplasmonics. Nature communications 2015, 6, 7132.

(92) Butet, J.; Dutta-Gupta, S.; Martin, O. J. Surface secondharmonic generation from coupled spherical plasmonic nanoparticles: Eigenmode analysis and symmetry properties. Physical Review B 2014, 89, 245449. 


\section{Graphical TOC Entry}

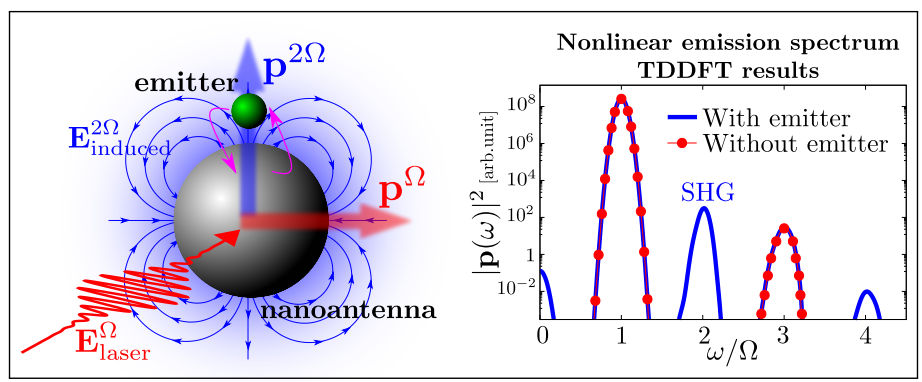

